\title{
Simulations of galactic disks including a dark baryonic component
}

\author{
Y. Revaz ${ }^{1,2}$, D. Pfenniger ${ }^{3}$, F. Combes ${ }^{2}$, and F. Bournaud ${ }^{4}$
}

${ }^{1}$ Laboratoire d'Astrophysique, École Polytechnique Fédérale de Lausanne (EPFL), 1290 Sauverny, Switzerland
e-mail: yves.revaz@epfl.ch
2 LERMA, Observatoire de Paris, 61 Av. de l'Observatoire, 75014 Paris, France
3 Geneva Observatory, University of Geneva, 1290 Sauverny, Switzerland
${ }^{4}$ Laboratoire AIM, CEA-Saclay DSM/DAPNIA/SAp-CNRS-Université Paris Diderot, 91191 Gif-Sur-Yvette, France

Received 1 April 2008 / Accepted 4 April 2009

ABSTRACT

\begin{abstract}
The near proportionality between $\mathrm{HI}$ and dark matter in outer galactic disks prompted us to run $\mathrm{N}$-body simulations of galactic disks in which the observed gas content is supplemented by a dark gas component representing between zero and five times the visible gas content. While adding baryons in the disk of galaxies may solve some issues, it poses the problem of disk stability. We show that the global stability is ensured if the ISM is multiphased, composed of two partially coupled phases, a visible warm gas phase and a weakly collisionless cold dark phase corresponding to a fraction of the unseen baryons. The phases are subject to stellar and UV background heating and gas cooling, and their transformation into each other is studied as a function of the coupling strength. This new model, which still possesses a dark matter halo, fits the rotation curves as well as the classical CDM halos, but is the only one to explain the existence of an open and contrasting spiral structure, as observed in the outer HI disks
\end{abstract}

Key words. galaxies: evolution - galaxies: ISM - galaxies: structure - galaxies: general - galaxies: kinematics and dynamics

\section{Introduction}

The $\Lambda \mathrm{CDM}$ scenario encounters much success in reproducing the large scale structures of the Universe traced by the Lyman- $\alpha$ forest and gravitational lensing (Springel et al. 2006). However, at galactic scale, where the baryonic physics plays a major role, the $\Lambda \mathrm{CDM}$ scenario has several well known problems. The dark matter cusp in galaxies (Blais-Ouellette et al. 2001; de Blok \& Bosma 2002; Swaters et al. 2003; Gentile et al. 2004, 2005; Spekkens et al. 2005; de Blok 2005; de Blok et al. 2008; Spano et al. 2008), and in particular in dwarf irregulars, the high number of small systems orbiting halos (Moore et al. 1999; Klypin et al. 1999; Strigari et al. 2007), the low angular momentum problem at the origin of too small disks (Navarro \& Benz 1991; Navarro \& Steinmetz 1997; Kaufmann et al. 2007), as well as the difficulty of forming bulgeless disks (Mayer et al. 2008) suggest that some physics is missing.

A strong prediction of the $\Lambda \mathrm{CDM}$ scenario is that the so called "missing baryons" reside in a warm-hot gas phase in the over-dense cosmic filaments (Cen \& Ostriker 1999, 2006). However, there are now several theoretical and observational arguments that support the fact that galactic disks may be more massive than usually thought, containing a substantial fraction of these "missing baryons".

It has been pointed out by Bosma $(1978,1981)$ that in samples of galaxies, the ratio between the dark matter and HI surface density is roughly constant well after the optical disk (see also Carignan et al. 1990; Broeils 1992; Hoekstra et al. 2001). This correlation may be a direct consequence of the conservation of the specific angular momentum of the gas during the galaxy formation process (Seiden et al. 1984). However, as shown by van den Bosch (2001) the angular momentum conservation leads to the formation of too concentrated stellar disks, a real problem for the low surface brightness galaxies. On the other hand, the HI-dark matter correlation may suggest that a large fraction of the dark matter lies in the disk of galaxies, following the HI distribution. This physical link between HI and dark matter has been confirmed by the baryonic Tully-Fischer relation of spiral galaxies (Pfenniger \& Revaz 2005) and more recently for extremely low mass galaxies (Begum et al. 2008).

Dark matter in the disk of galaxies is now also suggested by dynamical arguments based on the asymmetries of galaxies, either in the plane of the disks or transverse to it. For example, the large spiral structure present in the very extended HI disk of NGC 2915 is supported by a quasi self-gravitating disk (Bureau et al. 1999; Masset \& Bureau 2003). Revaz \& Pfenniger (2004) have shown that heavy disks are subject to vertical instabilities (also called bending instabilities) and may generate all types of observed warps: the common S-shaped but also the U-shaped and asymmetric ones (Reshetnikov \& Combes 1999; SánchezSaavedra et al. 2003). Other arguments for the origin of warps have been proposed (Jiang \& Binney 1999; Shen \& Sellwood 2006; Weinberg \& Blitz 2006), however none of them are able to explain the three types of warps.

Several works have tried to study the flattening of the Milky Way halo using potential tracers like dwarf galaxies (Johnston et al. 2005). From theses studies, the halo potential appears to be nearly spherical, with an ellipticity of 0.9 . However, dwarf galaxies trace the halo at distances larger than $20 \mathrm{kpc}$ where it is difficult to distinguish "classical" CDM disks with a slightly flattened halo from a heavy disk model, containing half of the halo mass in an extended flat disk (Revaz \& Pfenniger 2007).

A more accurate method is to trace the potential near the plane of the disk, using for example the vertical gas distribution. Kalberla (2003, 2004); Kalberla et al. (2007) have modeled the HI distribution out to a galactic radius of $40 \mathrm{kpc}$. Their self-consistent model is compatible with a self-gravitating dark matter disk having a mass of $2-3 \times 10^{11} M_{\odot}$. 
The presence of dark baryons in the disk of galaxies is reinforced by the numerous signs of recent star formation in the far outer disk of galaxies, correlated with HI gas in NGC 6946 (Ferguson et al. 1998), NGC 628 (Lelièvre \& Roy 2000), M 101 Smith et al. (2000), M 31 Cuillandre et al. (2001), NGC 6822 (de Blok \& Walter 2003), M 83 (Thilker et al. 2005). Correlations between young stars and HI far from the center reveal that molecular gas, at the origin of a weak but existing star formation rate, must be present in abundance there, despite the lack of $\mathrm{CO}$ detection.

Other arguments supporting the idea that dark matter could reside in the galactic disk in the form of cold molecular hydrogen $\left(\mathrm{H}_{2}\right)$ have been widely discussed by Pfenniger et al. (1994).

Unfortunately, a direct detection of cold $\mathrm{H}_{2}$ in the outer disk of galaxies appears to be a very hard task (Combes \& Pfenniger 1997). As the $\mathrm{H}_{2}$ molecule is symmetrical, any electric dipole moment is canceled. The molecule may be detected in emission only by its quadrupole radiation, the lowest corresponding to a temperature of $512 \mathrm{~K}$ above the fundamental state, much above the 5-7 K expected from Pfenniger \& Combes (1994). A weak radiation of the $\mathrm{H}_{2}$ ultra-fine lines is expected at kilometer wavelengths. However, since it is 10 orders of magnitude smaller than the HI line for the same density, its detection will not be possible in the near future. The absorption lines may be the best way to detect $\mathrm{H}_{2}$. But this method requires large statistics, as the filling factor of the gas is expected to be very low $(<1 \%)$.

The best tracers of cold $\mathrm{H}_{2}$ may be the pure rotational lines of $\mathrm{H}_{2}$ (at 28, 17, 12 and 9 microns), which could be emitted by a few percent of the molecular gas, excited by intermittent turbulence (see for example Boulanger et al. 2008).

Indirect detection by tracers may be prone to error. For example, $\mathrm{CO}$ traces the $\mathrm{H}_{2}$ but only for enriched gas and fails at large distances from galaxy centers. Moreover, it is impossible to detect $\mathrm{CO}$ emission from a cloud at a temperature close to the background temperature. The cold dust component detected by COBE/IRAS (Reach et al. 1995) is known to trace the cold $\mathrm{H}_{2}$. But as for the $\mathrm{CO}$, it is limited to small galactic radii where the cold gas is still mixed with some dust. Spitzer mid-infrared observations have recently revealed that large quantities of molecular hydrogen are not associated with star formation (Appleton et al. 2006; Ogle et al. 2007). An unexpectedly large amount of $10^{10} M_{\odot}$ of $\mathrm{H}_{2}$ is revealed only by its strong $\mathrm{H}_{2}$ emission lines in the galaxy Zwicky 3146 (Egami et al. 2006). Grenier et al. (2005) showed that much gas in the solar neighborhood is revealed only by $\gamma$-rays. Other indirect detections could be possible using micro-lensing events (Draine 1998; Fux 2005). However, this method has not been exploited up to now.

As direct and indirect observational detection of cold gas is difficult, it is necessary to test the effect a cold gas component would have on the global evolution of galactic disks. In this paper, we present new $N$-body simulations of galactic disks, where the observed gas content has been multiplied by a factor between 3 and 5. In addition to this extra dark baryonic component, a non-baryonic, spheroidal pressure-supported dark halo containing most of the large-scale dark mass is conserved.

An important issue that our model aims to answer is the stability question of heavy disks (Elmegreen 1997). Our model assumes that the additional baryons lie in a very cold and clumpy phase (Pfenniger \& Combes 1994), partially dynamically decoupled from the visible dissipative phase. We show that this phase can thus be less dissipative than the visible ISM and has larger velocity dispersions, so that the global disk stability is preserved. A new numerical implementation of the cycling acting between these two phases is proposed.
The secular evolution shows that the models with additional baryons are globally stable and share on average the same observational properties as the "classical" CDM disks. However, they give a natural explanation for the presence of contrasting spiral structures in the outskirts of HI disks which is difficult to explain when taking into account the self-gravity of the HI alone. In a forthcoming paper, we will show that this model also reproduces the puzzling dark matter content present in debris from galaxies (Bournaud et al. 2007).

Our model is different from previous multiphase models (Semelin \& Combes 2002; Harfst et al. 2006; Merlin \& Chiosi 2007) in the sense that it does not compute a cycling between a cold-warm dissipative and hot medium, but between a very cold weakly collisional phase and the visible dissipative phase.

The paper is organized as follows. Details of the multiphase model is given in Sect. 2. In Sect. 3 we briefly discuss the parameters used, and Sect. 4 is devoted to the galaxy model description. Section 5 compares the evolution of galaxy models with and without additional baryons and a short discussion. A summary is given in Sect. 6.

\section{The multiphase model}

\subsection{The straightforward approach}

The straightforward approach when modelling the galactic ISM is to assume that gas behaves like an ideal, inviscid gas. The evolution of the specific energy $u$ of the gas may be obtained by inserting the continuity equation into the first law of thermodynamics:

$\frac{\mathrm{d} u}{\mathrm{~d} t}=\frac{P}{\rho^{2}} \frac{\mathrm{d} \rho}{\mathrm{d} t}+\frac{\Gamma(\rho)-\Lambda(\rho, T)}{\rho}$.

The first right hand side term corresponds to the adiabatic behavior of the gas, while the second is responsible for the entropy variation. This latter reflects the non-adiabatic processes included through the heating and cooling function $\Gamma(\rho)$ and $\Lambda(\rho, T)$. At low temperature ISM $\left(T<10^{4} \mathrm{~K}\right)$, the heating is dominated by the cosmic ray heating (Goldsmith et al. 1969) and by the photo-electric UV heating on grains (Watson 1972; Draine 1978). As soon as the gas is enriched with metals, the cooling function is dominated by the CII, SiII, OI, FeII line emissions (Maio et al. 2007). Since the cooling function depends on the square of the gas density, the gas temperature is sensitive to it.

However, the ISM is known to be strongly non homogeneous down to very small scales, reaching densities higher than $10^{6}$ atoms $\mathrm{cm}^{-3}$ where the cooling time is very short, leading to equilibrium temperatures below $10 \mathrm{~K}$. Such over-densities are unfortunately far from being resolved by galactic scale simulations and numerical simulations miss the associated low temperatures. While being physically correct, Eq. (1) will then strongly bias the equilibrium temperature of the gas, because the estimated average density $\rho$ poorly reflects the actual physics.

\subsection{Statistical approach}

Instead of following the thermal specific energy of particles using the biased Eq. (1), we propose a new statistical approach avoiding the problem of the density and temperature evaluation. The multiphase ISM is assumed to be a two level system (see Fig. 1) with probabilities of transition depending only on the local excitation energy flux, that we call for short "UV flux", which is assumed to be the dominant heating process. 


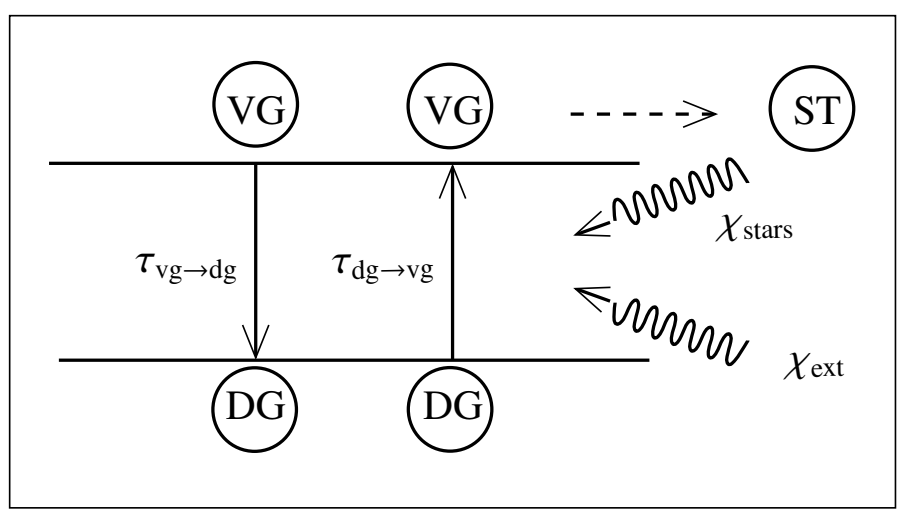

Fig. 1. Schematic representation of the ISM two levels system. The visible gas is labeled VG, the dark gas DG and the stars ST. The probability of transition between the visible and the dark gas depends on the UV flux generated by young stars $\left(\chi_{\star}\right)$ and from extragalactic sources $\left(\chi_{\text {ext }}\right)$ setting the characteristic transition times $\tau_{\mathrm{vg} \rightarrow \mathrm{dg}}$ and $\tau_{\mathrm{dg} \rightarrow \mathrm{vg}}$. Stars are formed out of the visible gas only.

The top level is populated by the well known observed dissipative gas detected by its $\mathrm{CO}, \mathrm{H}_{2}$ or $\mathrm{HI}$ emission. This phase will be called the visible gas. The bottom level is populated by undetected very clumpy and cold gas as proposed by Pfenniger \& Combes (1994), having temperature below the CO detection limit, at temperature equilibrium with the cosmic background radiation. This gas results from the strong cooling that occurs in overdense regions. As this gas is missed by all tracers, we will call it the dark gas.

According to the astrophysical literature, we have used here the word "gas". However, it is well known that the cold interstellar medium shows fractal properties which have been observed up to the instrumental capabilities, down to a few hundred AU (Heithausen 2004). Such heterogeneous fluids clearly do not have the viscosity or other mean properties of smooth flows. In other fields, it would be called granular flows, for example. We are aware of the degree of simplification of our model compared to the complexity of the ISM. A more complex model should include the dark component, the CO-undetected metalpoor warmer $\mathrm{H}_{2}$ gas that may exist in the outskirts of galactic disks (Papadopoulos et al. 2002), and possible effects related to phase transition and separation in the $\mathrm{He}-\mathrm{H}_{2}$ mixture at very cold temperature (Safa \& Pfenniger 2008).

The transition times between visible and dark gas is only dependent on the local UV flux $\chi$, which is the main heating process in the ISM. For the transition times, we have chosen the following simple relations:

$\left\{\begin{array}{l}\tau_{\mathrm{vg} \rightarrow \mathrm{dg}}=\chi^{+\beta} \tau, \\ \tau_{\mathrm{dg} \rightarrow \mathrm{vg}}=\chi^{-\beta} \tau,\end{array}\right.$

where $\beta$ and $\tau$ are two free parameters. $\tau$ gives the time scale of the transition, independently of the outer flux. $\beta$ sets the flux dependency. For $\beta=0$, the transition does not depend on the outer flux. At equilibrium, these equations provide simple relations for the ratio between the visible gas density $n_{\mathrm{vg}}$, the dark gas density $n_{\mathrm{dg}}$ and the total gas density $n_{\mathrm{tg}}$, as a function of the normalized local UV flux $\chi$ only $^{1}$ :

$\frac{n_{\mathrm{vg}}}{n_{\mathrm{tg}}}=\frac{\chi^{2 \beta}}{1+\chi^{2 \beta}}=\gamma$,

1 The solution is the one of a two level transition system with probabilities $p_{\mathrm{wc}} \sim 1 / \tau_{w c}$ and $p_{\mathrm{cw}} \sim 1 / \tau_{\mathrm{cw}}$. $\frac{n_{\mathrm{dg}}}{n_{\mathrm{tg}}}=\frac{1}{1+\chi^{2 \beta}}=1-\gamma$

$\frac{n_{\mathrm{dg}}}{n_{\mathrm{vg}}}=\chi^{-2 \beta}$

where in these latter equations, we have defined $\gamma$ as $n_{\mathrm{vg}} / n_{\mathrm{tg}}$. The interpretation of these equations is the following. When the gas is subject to a strong UV field, the heating dominates and the probability of transition between the dark and the visible gas is high. The gas is then dominated by the visible phase. On the contrary, when the UV field is very low (as the case in the outer regions of the galactic disk), the cooling dominates and the probability of a transition between the dark and the visible gas is low. The gas is then dominated by the dark phase.

\subsection{Visible and dark gas dynamics}

The visible phase is assumed to be dissipative, because it is more diffuse and collisional than the cold phase. Instead of using the classical SPH approach, which makes the gas strongly collisional, we have preferred to use the sticky particle scheme (Brahic 1977; Schwarz 1981; Combes \& Gerin 1985) which better simulates the clumpy ISM. In the following simulations, we have used $\beta_{\mathrm{r}}=0.8$ and $\beta_{\mathrm{t}}=1$ in order to strictly conserve angular momentum ${ }^{2}$. The frequency of collisions between particles is set to be proportional to the local visible gas density.

Also, as the very cold and clumpy dark gas does not radiate, it is expected to be weakly collisional. Its relaxation time being much longer than the dynamical time, we neglect here the effect of collisions.

\subsection{Star formation}

Stars that are assumed to be the main source of UV flux in the inner part of the galaxy may be formed out of the visible gas only. We have used a classical star formation recipe (Katz et al. 1996) that reproduces well the Schmidt law.

\subsection{UV flux}

The normalized UV energy flux $\chi$ is decomposed into two parts. A stellar radiation flux $\chi_{\star}$ and an extragalactic background energy flux $\chi$ ext.

\subsubsection{Stellar UV flux}

The stellar normalized radiation flux is computed by summing the contribution of each star, assuming an appropriate $(L / M)$ ratio.

$\chi_{\star}=\frac{1}{4 \pi c U_{\odot}} \sum \frac{m_{i}(L / M)_{i}}{r_{i}^{2}+\epsilon^{2}}$,

where $m_{i}$ is the particle of mass $i$ and $r_{i}$ its distance to the point where the flux is estimated. $\epsilon$ is the gravitational softening, $c$ the speed of light and $U_{\odot}$ is the Habing UV energy density equal to $5.4 \times 10^{-14} \mathrm{erg} / \mathrm{cm}^{3}$ (Habing 1968). The ratio $(L / M)$ is set to $1.58 \times 10^{31} \mathrm{erg} / \mathrm{s} / M_{\odot}$. This value is constant for all particles (independently of the mass and type of star particles). The summation in Eq. (6) is performed over all star particles and may be very time consuming in a large $N$-body system. We have computed it

\footnotetext{
${ }^{2}$ See Bournaud \& Combes (2002) for details on the meaning of $\beta_{\mathrm{r}}$ and $\beta_{\mathrm{t}}$.
} 
Table 1. Multiphase model parameters.

\begin{tabular}{cccc}
\hline \hline$\beta$ & $(L / M)$ & $U_{\text {ext }}$ & $\tau$ \\
& {$\left[\mathrm{erg} / \mathrm{s} / M_{\odot}\right]$} & {$\left[10^{11} M_{\odot}\right]$} & {$[\mathrm{Gyr}]$} \\
\hline 1 & $1.58 \times 10^{32}$ & $1.8 \times 10^{14}$ & 10 \\
\hline
\end{tabular}

by taking advantage of its similarity to the summation of gravitational forces which is computed using the treecode method. The time overhead for the computation of $\chi_{\star}$ is then negligible.

\subsubsection{Extragalactic UV flux}

The extragalactic UV background normalized radiation flux is assumed to be constant.

$\chi_{\text {ext }}=\frac{U_{\text {ext }}}{U_{\odot}}$

where the extragalactic UV background density is set to $2 \times$ $10^{-15} \mathrm{erg} / \mathrm{cm}^{3}$.

\section{Parameters}

Our multiphase model is based on 4 parameters. The external energy density flux $U_{\text {ext }}$, the UV light to mass ratio $(L / M)$, the coefficient parameter $\beta$ and the time scale of transition $\tau$. In this paper, we have fixed the 4 parameters to constant values given in Table 1 . The parameter $\beta$ is set to 1 for simplicity. The parameter $(L / M)$ is chosen such that the normalized stellar UV flux $\chi_{\star}$ is unity near $15 \mathrm{kpc}$ when the stellar distribution at the origin of the UV flux corresponds to a realistic exponential disk, as will be presented in Sect. 4. According to Eq. (5), these values correspond to the radius where the dark component surface density is equivalent to the visible surface density. $U_{\text {ext }}$ is chosen such that, in the absence of a stellar UV field (at a distance $R=\infty$ from the galaxy center), the ratio $n_{\mathrm{vg}} / n_{\mathrm{vg}}=\gamma_{\infty}$ where $\gamma_{\infty}$ has been set to $1 / 10$. From Eq. (3) and setting $\chi_{\star}=0$ we get:

$U_{\text {ext }}=U_{\odot}\left(\frac{\gamma_{\infty}}{1-\gamma_{\infty}}\right)^{\frac{1}{2 \beta}}$.

The time scale of the transition $\tau$ is set to about 10 dynamical times so that the cycling between visible and dark gas is slow. Figure 2 shows the total normalized flux $\chi$ as well as the visible over total gas mass ratio $\gamma$ obtained with the parameters of Table 1 and the stellar distribution of Sect. 4. As an illustration, the flux is applied on a purely exponential baryonic gaseous disk. The resulting surface densities for the visible and dark gaseous disk are displayed in the bottom of the figure. The exponential disk generates a strong UV flux at the center. Consequently, the visible gas dominates there. On the contrary, in the outer part, the UV flux is dominated by the constant weak extragalactic flux, making the dark gas dominant.

\section{Galaxy models}

We have used our multiphase model to simulate the evolution of Milky Way-like spiral galaxies. In order to understand the effect of the multiphase model, we have compared two models, including additional dark baryons, with a reference "classical" model. In addition, we have build a fourth simulation including the perturbation of $\Lambda \mathrm{CDM}$ substructures. In the following sections, we first present our reference model (also called $N=1$

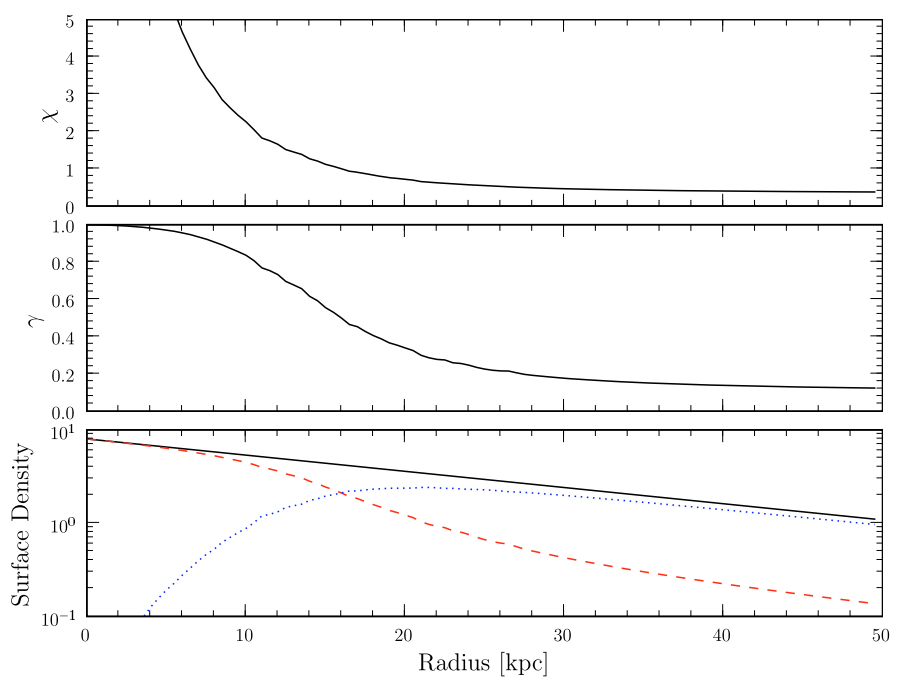

Fig. 2. Normalized UV flux $\chi$, visible to total gas density ratio $\gamma$ and surface density of the gas, as a function of the galactic radius $R$. In the bottom graph, the red dashed line corresponds to the visible gas, the blue dotted line to the dark gas and the dark plain line to the total gas.

model), where no additional dark baryons have been added. In the next section (Sect. 4.1.2) we present how it is possible to add dark baryons in the reference model, in a way consistent with the rules proposed in Chapter 2 and by conserving the same observational properties of the reference model. The model including $\Lambda \mathrm{CDM}$ substructures will be presented in Sect. 4.1.3. Section 4.2 will discuss how initial velocities have been set in order to make the disk stable.

\subsection{Mass distribution}

\subsubsection{The reference model}

Our reference model is designed to fit typical properties of giant Sbc galaxies with a flat extended rotation curve. It is initially composed of a bulge, an exponential stellar disk, a gas disk and a dark matter halo.

1. The bulge density profile is a simple Plummer model:

$$
\rho^{\mathrm{b}}(R, z) \propto\left(1+\frac{r^{2}}{r_{\mathrm{b}}^{2}}\right)^{-5 / 2},
$$

with a characteristic radius $r_{\mathrm{b}}=1.4 \mathrm{kpc}$.

2. The exponential stellar disk takes the usual form:

$\rho^{\mathrm{d}}(R, z) \propto \mathrm{e}^{-R / H_{R}} \mathrm{e}^{-|z| / H_{z}}$,

where the radial and vertical scale length are respectively $H_{R}=4$ and $H_{z}=0.3 \mathrm{kpc}$.

3. The dark matter is distributed in a Plummer model:

$$
\rho^{\mathrm{h}}(R, z) \propto \rho_{\left(0, r_{\mathrm{dm}}\right)}^{\mathrm{MN}},
$$

with a characteristic radius $r_{\mathrm{dm}}=30 \mathrm{kpc}$, ensuring a flat rotation curve up to $40 \mathrm{kpc}$. The model is truncated at $3 r_{\mathrm{dm}}$ $(90 \mathrm{kpc})$. In order to avoid any perturbation of the halo on the disk due to imperfect equilibrium, we have set it as a rigid potential. We have checked that a live halo will not influence our results.

4. The choice of the visible gas distribution follows the observations of Hoekstra et al. (2001), where the dark matter 
Table 2. Parameters for the reference giant Sbc model.

\begin{tabular}{llcc}
\hline \hline Component & Model & $\begin{array}{c}\text { Parameters } \\
{[\mathrm{kpc}]}\end{array}$ & $\begin{array}{c}\text { Mass } \\
{\left[10^{11} M_{\odot}\right]}\end{array}$ \\
\hline bulge & Plummer & $r_{\mathrm{b}}=1.4$ & 0.28 \\
disk & exponential & $H_{R}=4, H_{z}=0.3$ & 0.69 \\
halo & Plummer & $r_{\mathrm{dm}}=30$ & 5.17 \\
gas & Miyamoto-Nagai & $a=30, b=0.3$ & 0.21 \\
\hline
\end{tabular}

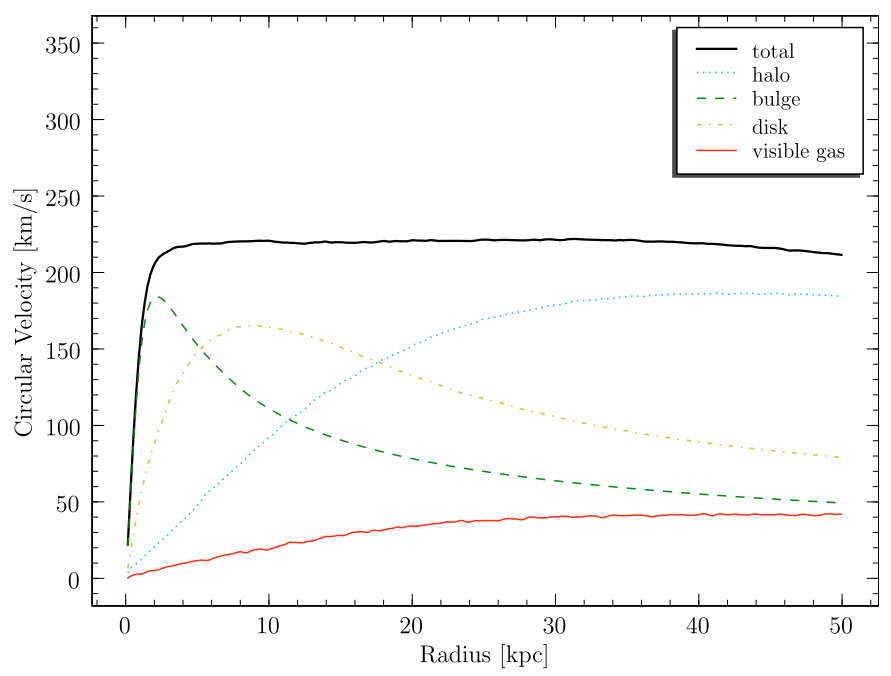

Fig. 3. Rotation curve of the reference model with the contribution of each component, the bulge, the exponential disk, the visible gas and the dark matter halo.

contribution to the rotation curve is a multiple of the contribution of the gas. This is achieved by distributing the gas in a Miyamoto-Nagai model (Miyamoto \& Nagai 1975):

$\rho^{\mathrm{vg}}(R, z) \propto \rho_{\left(h_{z}, r_{\mathrm{dm}}\right)}^{\mathrm{MN}}$.

The proportionality between the Plummer halo (being in fact a subclass of a Miyamoto-Nagai model) and the disk is ensured if the disk scale length is similar to the one of the halo: $h_{R}=r_{\mathrm{dm}}$ (see Appendix A). The vertical scale height $h_{z}$ is fixed to $0.3 \mathrm{kpc}$. We also have included a flaring of the visible gas disk by multiplying the $z$ coordinates of the particles by:

$\exp \left(\frac{R}{R_{\mathrm{f}}}\right)$

where the transition radius $R_{\mathrm{f}}$ is set to $40 \mathrm{kpc}$.

Mass and parameters of the model are summarised in Table 2 and the corresponding rotation curve is displayed in Fig. 3.

\subsubsection{Adding dark baryons in the disk}

Here, we present a method that allows us to build new galaxy models containing additional dark baryons, but presenting similar observational properties as the reference model. The new models are build by following three rules:

1. the total rotation curve remains nearly unchanged with respect to the reference model;

2. the surface density of the visible gas remains nearly unchanged with respect to the reference model;

3. no dynamically significant dark matter is added in the central regions.
In addition to these rules, the model, must also respect the ratio between the visible and additional dark gas that follows from the multiphase model proposed in Sect. 2.

The third rule is derived from the luminosity Milky-Way model (Bissantz \& Gerhard 2002) combined with MACHO micro-lensing observations (see Gerhard 2006, and references therein for more details). We thus assume 1) no dark gas exists at the center of galaxies, and 2) the density of the total gas ( $\mathrm{tg}$ ) is equal to that of the visible gas (vg):

$\rho_{\text {in }}^{\mathrm{tg}}=\rho^{\mathrm{vg}}$, in the central regions.

This equation also ensures the second rule at the center.

In the outer part, the total gas mass distribution is constrained by the first rule. We can easily transfer mass from the halo to the disk without changing the rotation curve, using the equivalence in term of density of Eq. (A.5) (via the Poisson equation). In that case, the density of the halo plus the total gas (htg) (including visible and dark gas) may be written as:

$\rho_{M_{\mathrm{htg}}}^{\mathrm{htg}}=f \rho_{\left(M_{\mathrm{htg}}, h_{z}, r_{\mathrm{dm}}\right)}^{\mathrm{MN}}+(1-f) \rho_{\left(M_{\mathrm{htg},}, 0, r_{\mathrm{dm}}\right)}^{\mathrm{MN}}$,

where the first left side term corresponds to the total gas density:

$\rho_{M_{\mathrm{tg}}, \mathrm{out}}^{\mathrm{tg}}=f \rho_{\left(M_{\mathrm{htg}}, h_{z}, r_{\mathrm{dm}}\right)}^{\mathrm{MN}}$,

and the second left side term corresponds to the halo density:

$\rho_{M_{\mathrm{h}}}^{\mathrm{h}}=(1-f) \rho_{\left(M_{\left.\mathrm{htg}, 0, r_{\mathrm{dm}}\right)}^{\mathrm{MN}} .\right.}^{\mathrm{M}}$

In this equation, the mass of each component is given as an index. $M_{\text {htg }}$ is the sum of the halo and gas mass of the reference model. $M_{\mathrm{tg}}=f M_{\mathrm{htg}}$ is the total gas mass and $M_{\mathrm{h}}=(1-f) M_{\mathrm{htg}}$ is the halo mass. It is convenient to define $f$ as:

$f=N \frac{M_{\mathrm{g}}}{M_{\mathrm{htg}}}$,

where $N$ gives the ratio between the total gas and the gas of the reference model. Its value is restricted to the range $\left[0, M_{\mathrm{htg}} / M_{\mathrm{g}}\right]$. When $N=1$, Eqs. (16) and (17) give the densities corresponding to the reference model.

We can derive the total gas density all along the disk by combining Eqs. (14) and (16):

$$
\begin{aligned}
\rho^{\operatorname{tg}}(R) & =\alpha(R) \rho_{\mathrm{in}}^{\mathrm{tg}}(R)+(1-\alpha(R)) \rho_{\mathrm{out}}^{\mathrm{tg}}(R) \\
& =\alpha(R) \rho^{\mathrm{vg}}(R)+(1-\alpha(R)) f \rho_{\left(M_{\mathrm{htg}}, h_{z}, r_{\mathrm{dm}}\right)}^{\mathrm{MN}}(R),
\end{aligned}
$$

where we have introduced a new function $\alpha(R)$ taking values of 1 at the center and 0 in the outer part of the disk.

We now introduce the multiphase model of Sect. 2 that places constraints on the ratio between visible and dark gas. Applying Eq. (4) to the total gas density $\rho_{\text {tg }}$ we can derive the density of the visible gas:

$\rho^{\mathrm{vg}}(R)=\gamma(R) \rho^{\operatorname{tg}}(R) \quad$ where $\quad \gamma(\mathrm{R})=\frac{\chi^{2 \beta}}{1+\chi^{2 \beta}}$,

and where $\gamma(R)$ (see Eq. (4)) follows from the stellar mass distribution, the $(L / M)$ ratio and the UV background. Combining Eqs. (20) and (21) allows us to determine the value of $\alpha(R)$ which completely defines the total gas distribution:

$\alpha(R)=\frac{1}{\gamma(R)}\left(\frac{\gamma(R) f \rho_{\left(M_{\mathrm{htg}}, h_{z}, r_{\mathrm{dm}}\right)}^{\mathrm{MN}}(R)-\rho^{\mathrm{vg}}(R)}{f \rho_{\left(M_{\mathrm{htg}}, h_{z}, r_{\mathrm{dm}}\right)}^{\mathrm{MN}}(R)-\rho^{\mathrm{vg}}(R)}\right)$. 
Table 3. Parameters for the reference giant Sbc model.

\begin{tabular}{cccccc}
\hline \hline$N$ & $\begin{array}{c}M_{\mathrm{vg}} \\
{\left[10^{11} M_{\odot}\right]}\end{array}$ & $\begin{array}{c}M_{\mathrm{dg}} \\
{\left[10^{11} M_{\odot}\right]}\end{array}$ & $\begin{array}{c}M_{\mathrm{b}} \\
{\left[10^{11} M_{\odot}\right]}\end{array}$ & $\begin{array}{c}M_{\text {Tot }} \\
{\left[10^{11} M_{\odot}\right]}\end{array}$ & $\begin{array}{c}M_{\mathrm{b}} / M_{\text {Tot }} \\
\%\end{array}$ \\
\hline 1 & 0.21 & 0.00 & 1.18 & 6.36 & 18 \\
3 & 0.12 & 0.42 & 1.52 & 6.27 & 24 \\
5 & 0.16 & 0.66 & 1.80 & 6.12 & 29 \\
\hline
\end{tabular}

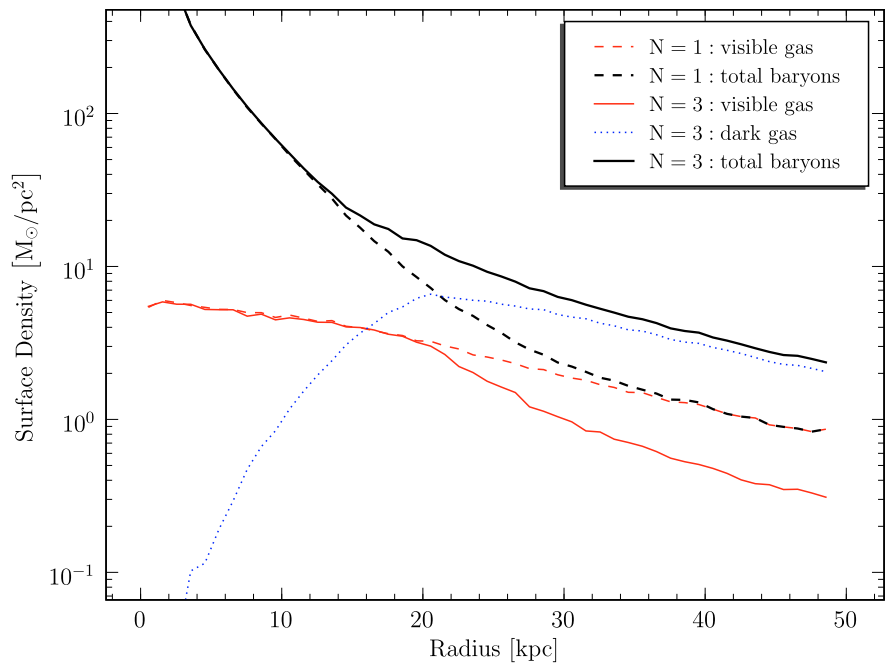

Fig. 4. Comparison of the surface density of baryons between model $N=1$ and model $N=3$. The total baryons (including stars) are traced in black. The red line represents the visible gas while the blue line (only present for the $N=3$ model) falling towards the center corresponds to the dark gas.

In this equation, we force values of $\alpha$ to stay in the range [0, 1]. As we will see later, this restriction breaks the second rule in the outer part of the disk. Finally, the same flaring as for the visible gas (Eq. (13)) is applied to the total gas.

Following this scheme, we have constructed two different models, with respectively $N=3$ and $N=5$. Applying the multiphase model with parameters listed in Table 1, we can now determine the mass properties of the different components (visible gas, dark gas, total baryons and dark halo) in these models, including the reference model $(N=1)$, given in Table 3 .

A larger value of $N$ increases the dark mass and inversely decreases the dark halo mass (the total mass remaining constant). The baryon fraction $\left(M_{\mathrm{b}} / M_{\mathrm{tot}}\right)$ is then an increasing function of $N$ and grows from the universal baryonic fraction up to $30 \%$ for model $N=5$. The total visible mass is not strictly identical between the three models. Its variation is mainly due to the the outer part where its surface density decreases with respect to the reference model. However, the visible gas lying below $20 \mathrm{kpc}$ is similar for the 3 models $\left(M_{\mathrm{vg}}=5.4 \times 10^{9} M_{\odot}\right)$.

The gas and total baryon surface density is displayed in Figs. 4 and 5. The outer regions $(R>20 \mathrm{kpc})$ of models $N=3$ and $N=5$ are characterised by the presence of the dark gas which dominates the surface density of the baryons. In the far outer parts, the baryons are multiplied by $N$, with respect to the reference model. On the contrary, due to the drop in the dark gas surface density, the visible gas surface density of the inner regions is left unchanged with respect to the reference model (second rule). The presence of dark gas is only marginal inside $10 \mathrm{kpc}$.

Figures 6 and 7 compare the contribution of all components to the rotation curve. In the models with additional baryons, the decreasing contribution of the dark halo mass in the outer part

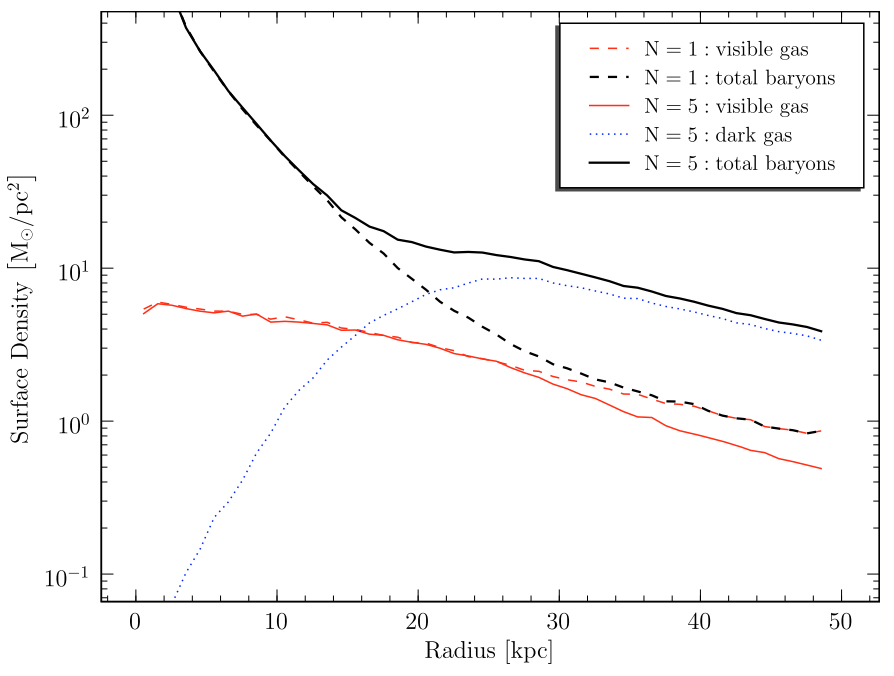

Fig. 5. Same figure as Fig. 4 but for model $N=5$.

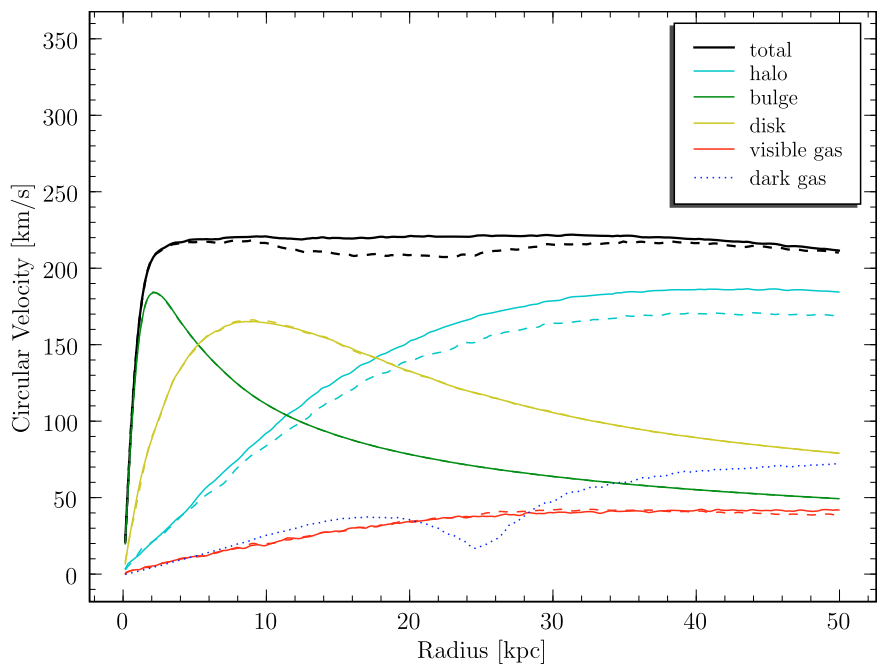

Fig. 6. Comparison of the rotation curves of model $N=1$ and model $N=3$ and the contribution of each component.

is compensated by the dark gas, ensuring a flat rotation curve up to $50 \mathrm{kpc}$. The bump in this component, appearing below $25 \mathrm{kpc}$, is due to the fact that, as no dark gas resides in the central regions, the square velocity of this component alone is negative. As in Figs. 6 and 7 we have plotted the absolute value of the velocity, the imaginary part appears as positive. Except around the transition radius of $20 \mathrm{kpc}$, the total rotation curve of models $N=3$ and $N=5$ remains nearly unchanged with respect to the reference model (first rule).

\subsection{3. $\Lambda$ CDM substructures}

In addition to the three previous models, we have built a fourth model called the $N=1+s$ model based on the $N=1$ models but taking into account a sample of dark matter satellites predicted by the $\Lambda$ CDM cosmology, orbiting around the disk and interacting with it.

The purpose of this model is to study the effect of $\Lambda \mathrm{CDM}$ satellites on the spiral structure of the disk, and to compare it with the effect of the additional baryons taken into account in models $N=3$ and $N=5$. 


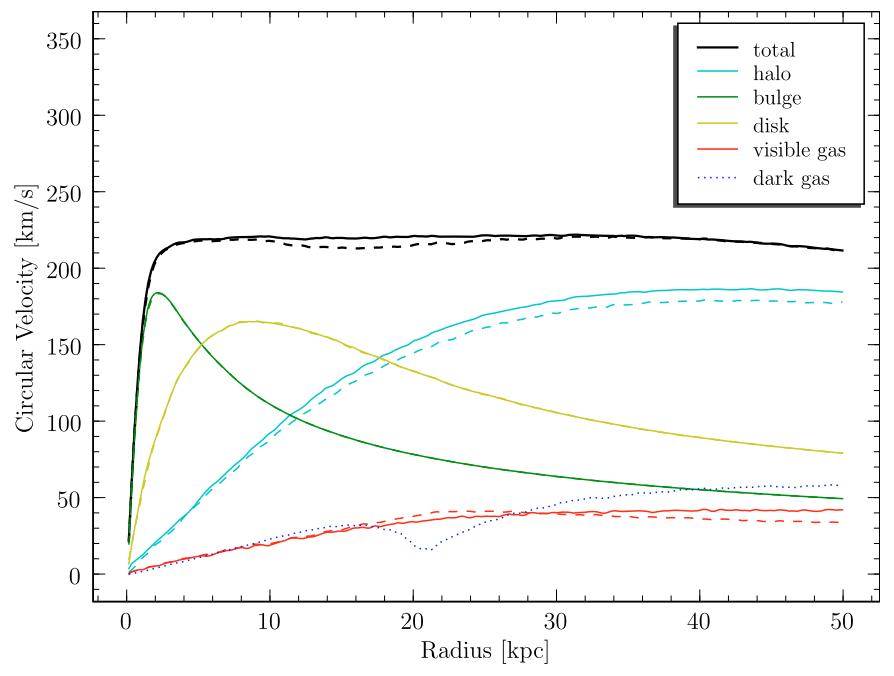

Fig. 7. Same figure as Fig. 6 but for model $N=5$.

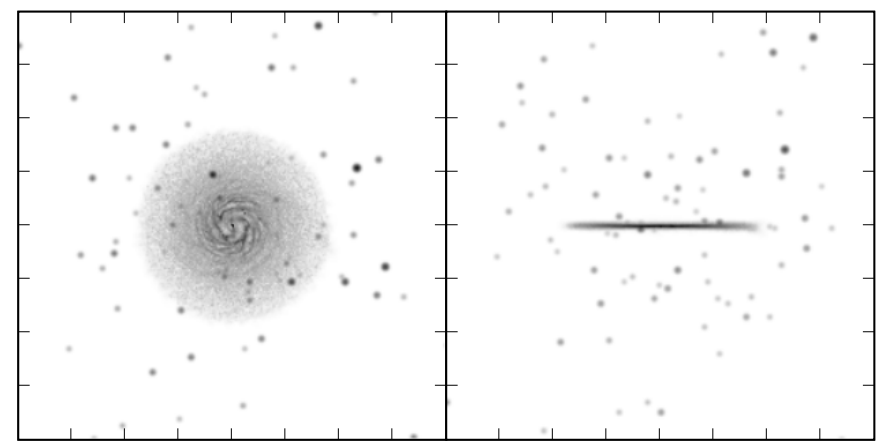

Fig. 8. Model $N=1+s$ : $100 \Lambda \mathrm{CDM}$ satellites around the gaseous disk of the $N=1$ model. The images have been take after $t=2.2 \mathrm{Gyr}$ of evolution. The box size is $400 \times 400 \mathrm{kpc}$.

The $\Lambda \mathrm{CDM}$ satellites have been added following the technique described by Gauthier et al. (2006) based on the distribution function of Gao et al. (2004), in agreement with recent $\Lambda C D M$ simulations. 100 satellites extending up to $250 \mathrm{kpc}$, with masses between $1.5 \times 10^{-4}$ and 0.02 mass of the galaxy of the reference model $\left(6.36 \times 10^{11} M_{\odot}\right)$, have been added, representing $10 \%$ of the total galactic mass. Contrary to Gauthier et al. (2006), in our simulations, the substructures are Plummer masses with a softening fixed to $0.25 \mathrm{kpc}$. Figure 8 shows the surface density of model $N=1+s$ including the $\Lambda$ CDM satellites. The darkness of the satellites scales as a function of the mass.

\subsection{Velocity distribution}

The velocity distributions are computed following the method proposed by Hernquist (1993). For the spherical distribution, the bulge, the velocity dispersion is assumed to be isotropic and is derived from the second moment of the Jeans equation (Binney \& Tremaine 1987) which is then:

$\sigma_{\mathrm{h}}^{2}(r)=\frac{1}{\rho_{\mathrm{h}}(r)} \int_{r}^{\infty} \mathrm{d} r^{\prime} \rho_{\mathrm{h}}\left(r^{\prime}\right) \partial_{r^{\prime}} \Phi\left(r^{\prime}\right)$.

For the axisymmetric components (stellar disk and gas disk), we first compute the vertical velocity dispersion $\sigma_{z}$, by satisfying the Jeans equation in cylindrical coordinates (Binney $\&$ Tremaine 1987) separately for each component $i$ :

$\sigma_{z i}^{2}=\frac{1}{\rho_{i}(z)} \int_{z}^{\infty} \mathrm{d} z^{\prime} \rho_{i}\left(z^{\prime}\right) \partial_{z^{\prime}} \Phi\left(z^{\prime}\right)$.

For the stellar disk and for the bulge, the radial velocity dispersion $\sigma_{R}$ is fixed by imposing the value of the Safronov-Toomre parameter $Q$ to 1 , ensuring the horizontal stability of the disk. $Q$ is defined by:

$$
Q=\frac{\kappa \sigma_{R}}{3.36 G\left(\Sigma_{d}+\Sigma_{g}\right)}
$$

where $\kappa$ is the radial epicyclic frequency. For the gas, according the the HI observations, $\sigma_{R}$ is fixed to a value of $10 \mathrm{~km} \mathrm{~s}^{-1}$. The tangential velocity $\sigma_{\phi}$ is derived from the epicycle approximation (Binney \& Tremaine 1987, p. 125):

$$
\frac{\sigma_{\phi}^{2}}{\sigma_{R}^{2}}=\frac{\kappa^{2}}{4 \Omega^{2}}=\frac{R^{-3} \partial_{R}\left(R^{3} \partial_{R} \Phi\right)_{z=0}}{4 R^{-1}\left(\partial_{R} \Phi\right)_{z=0}},
$$

where the rotation frequency $\Omega$ is determined from the potential $R$-derivatives as indicated in Eq. (26). Finally, the mean azimuthal velocity is deduced from the second moment of the Jeans equation:

$\left\langle v_{\phi}^{2}\right\rangle=R \partial_{R} \Phi(R, z)+\sigma_{R}^{2}-\sigma_{\phi}^{2}+\frac{R}{\rho_{i}} \partial_{R}\left(\rho_{i} \sigma_{R}^{2}\right)$,

where we have assumed that the term $\left\langle v_{R} v_{\phi}\right\rangle=0$.

\subsubsection{Velocity distribution for the visible and dark gas}

The radial velocity dispersions of the visible gas is set to be constant at $10 \mathrm{~km} \mathrm{~s}^{-1}$ as observed:

$\sigma_{R \mathrm{vg}}=10 \mathrm{~km} \mathrm{~s}^{-1}$.

On the contrary, in order to ensure stability, the radial velocity dispersions of the dark gas is set by imposing the SavronovToomre parameter to be 1:

$\sigma_{R \mathrm{dg}}=\frac{3.36 G\left(\Sigma_{d}+\Sigma_{g}\right) Q}{\kappa}, \quad$ with $\quad Q=1$.

The velocity dispersions and the mean azimuthal velocity are then used to distribute the model particles in the velocity space.

\section{Model evolution}

The multiphase model has been implemented on the parallel Tree code Gadget 2 (Springel 2005). The models $N=1, N=$ $1+s, N=3$ and $N=5$ contain respectively 319315,319415 , 535769 and 731129 particles. The mass of the gas particles is constant between the three models. The softening length is set to $250 \mathrm{pc}$. All simulations have been run between 0 and $4.5 \mathrm{Gyr}$. For simplicity, the feedback from supernovae has been turned off.

\subsection{Global properties}

Despite their different dark matter content, after $4.5 \mathrm{Gyr}$, the three models $(N=1, N=3$ and $N=5)$ still share similar global properties.

In Fig. 9 we compare the total velocity curve of the three models, after $4.5 \mathrm{Gyr}$ of evolution. In the central part, they all 


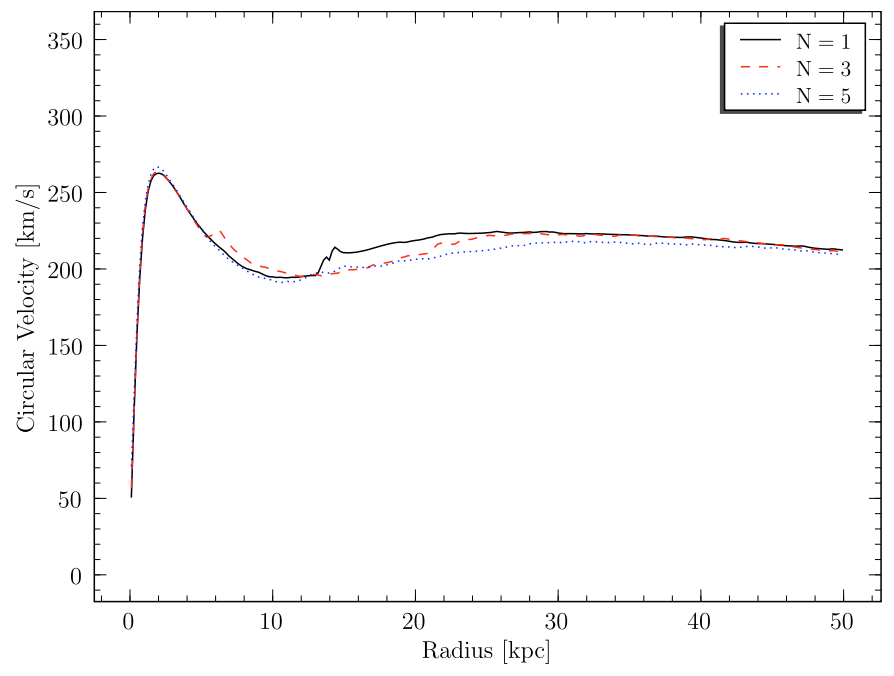

Fig. 9. Comparison of the rotation curve of the 3 models at $t=4.5 \mathrm{Gyr}$.

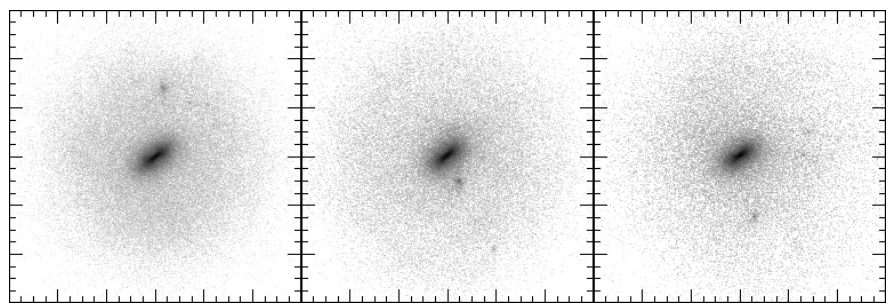

Fig. 10. Surface density map of the stellar disk of the 3 models $(N=1$, $N=3$ and $N=5$, from left to right) at $t=4.5 \mathrm{Gyr}$. The box size of each image is $60 \times 60 \mathrm{kpc}$.

show a bump corresponding to the presence of a bar. In the outer part, all curves converge to the same values. The main differences occur around the transition radius $(\sim 10-20 \mathrm{kpc})$, where models with additional baryons have slightly lower values. However, these differences are simply a relic of the differences existing in the initial conditions (see Figs. 6 and 7). The similarity of the rotation curves means that the three models share the same radial potential dependency, having similar horizontal epicyclic frequencies ( $\kappa$ and $\Omega$ ). The pattern speed, the shape and the extension of the bar is also identical (see Fig. 10).

The surface density of the three models is compared in Fig. 11. For radius smaller than $30 \mathrm{kpc}$, the curves corresponding to the visible gas surface density are superimposed. Differentiating these three curves is only possible in the far outer part, as was the case for the initial conditions (see Figs. 4 and 5). It is thus important to notice that the rotation curve and the azimuthal averaged surface density are not sufficient to easily distinguish between these three models, even after 4.5 Gyr of evolution.

In Fig. 12 we compare the scale height of the disk of the four models at $t=0.9$ and at $t=4.1 \mathrm{Gyr}$. The continuous lines correspond to the visible gas while the dotted ones to the dark gas. At $t=0.9 \mathrm{Gyr}$, no difference exists between the four models. After 4 Gyr of evolution the visible gas in the $N=1+s, N=3$ and $N=5$ models has been slightly heated and presents higher scale heights. In the case of model $N=1+s$, the increase of the scale height is attributed to the perturbation of the $\Lambda$ CDM satellites (Font et al. 2001; Dubinski et al. 2008), while for models $N=3$ and $N=5$ it is due to the coupling with the collisionless dark gas that has a higher scale height (dotted lines). From an

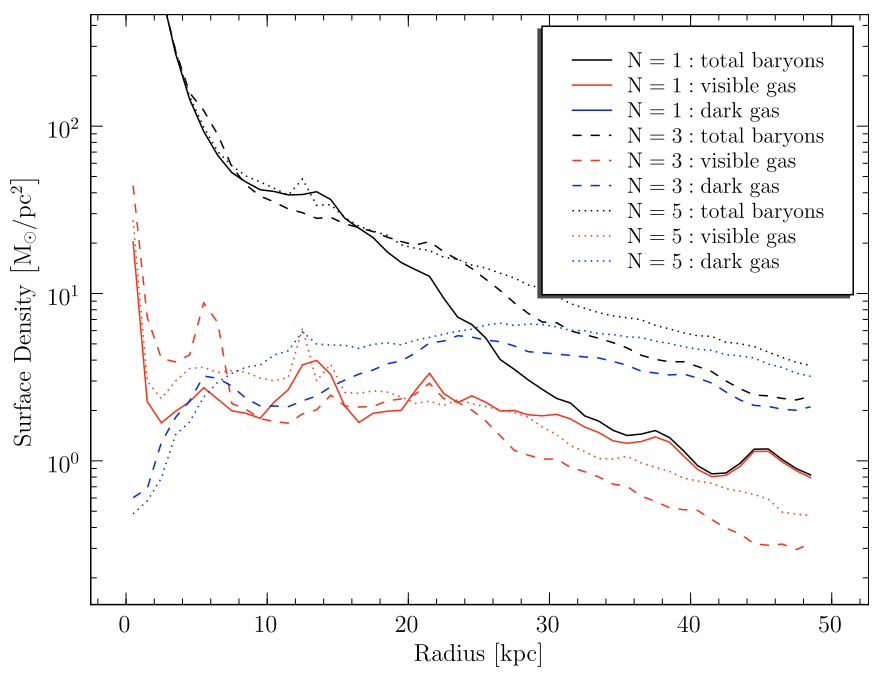

Fig. 11. Comparison of the surface density of different components at $t=4.5 \mathrm{Gyr}$.

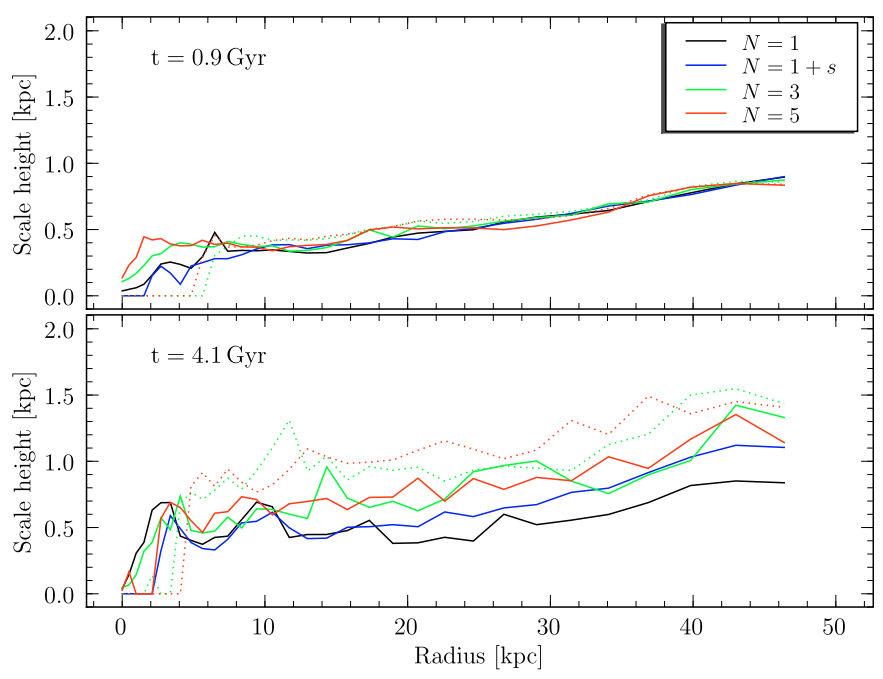

Fig. 12. Disk scale height as a function of the radius, for the four models at $t=0.9$ and $t=4.1$ Gyr. The lines corresponds to the visible gas while the dotted lines correspond to the dark gas.

observational point of view, models $N=1+s, N=3$ and $N=5$ will be very similar.

\subsection{Disk stability and spiral structures}

The main differences between the models with additional dark baryons and the "classical model" appear when comparing the spiral structure of the visible gas. The surface density evolution of the three models plus the one with the $\Lambda$ CDM satellites is displayed in Figs. 13-16. In the two latter plots, in addition to the visible gas surface density, the dark gas surface density is also displayed. These plots emphasize two important points. First, despite the presence of larger amount of baryons in the outer parts, the disks of models $N=3$ and $N=5$ are globally stable over the 4.5 simulated Gyrs. Secondly, spiral structures have very different patterns and may be used to distinguish between models with and without additional baryons.

At all times during the evolution, the surface density of the dark gas decreases at the center, preserving a hole. This hole 


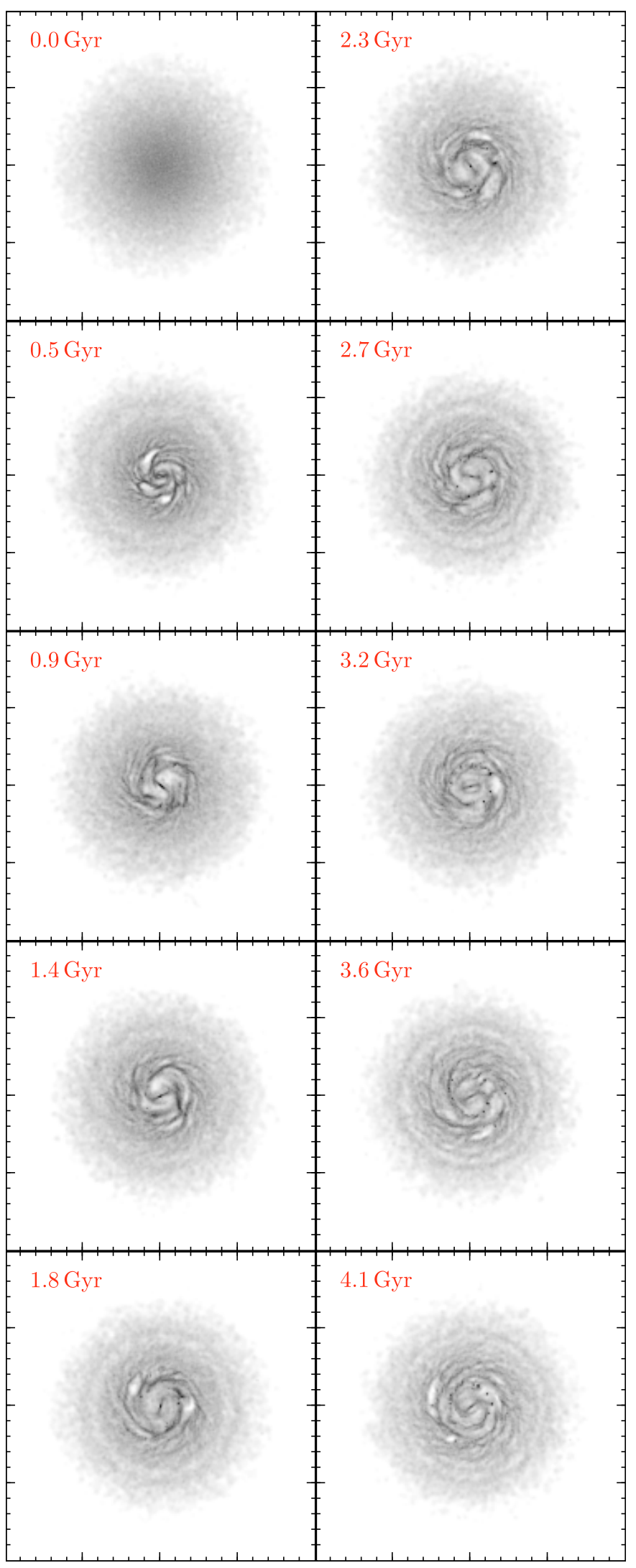

Fig. 13. Evolution of model $N=1$ between 0 and 4.5 Gyr. Each image represents the surface density of the visible gas. The box size is $200 \times$ $200 \mathrm{kpc}$.

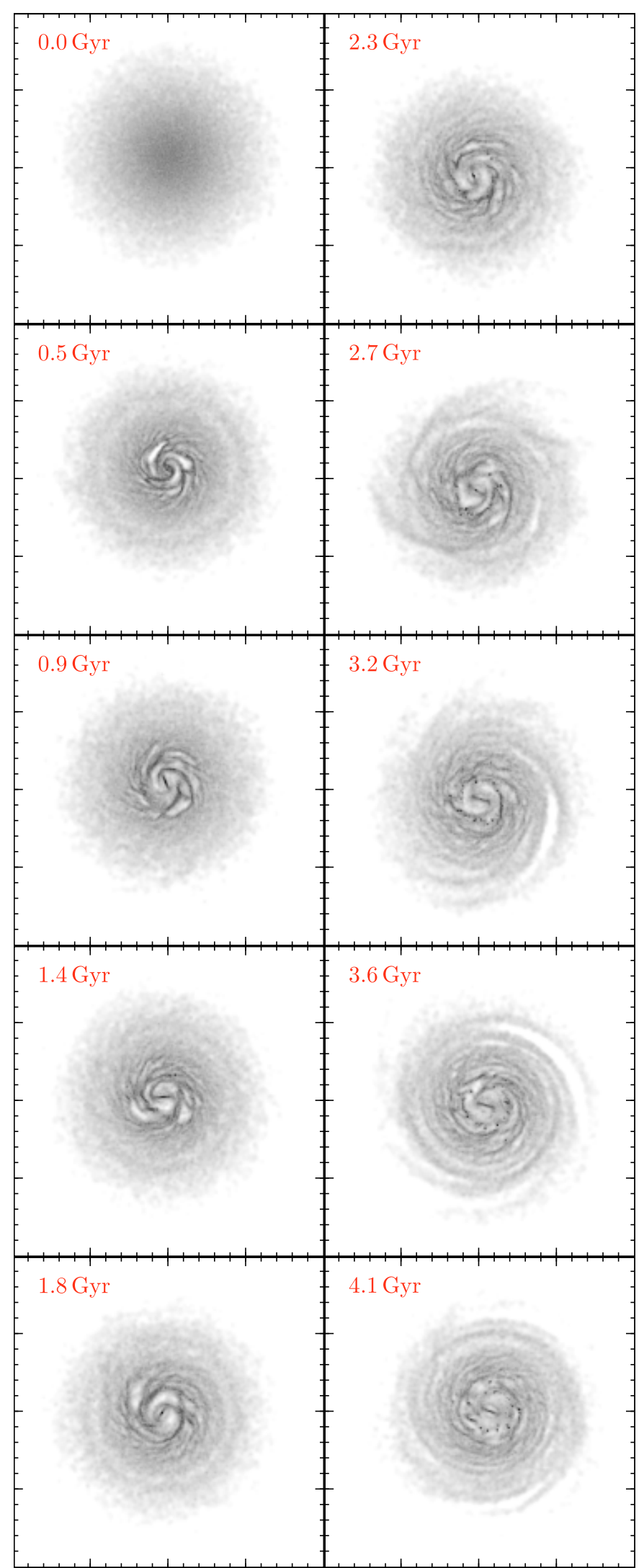

Fig. 14. Evolution of model $N=1$ including $100 \Lambda \mathrm{CDM}$ substructures. Each image represents the surface density of the visible gas. The box size is $200 \times 200 \mathrm{kpc}$. 


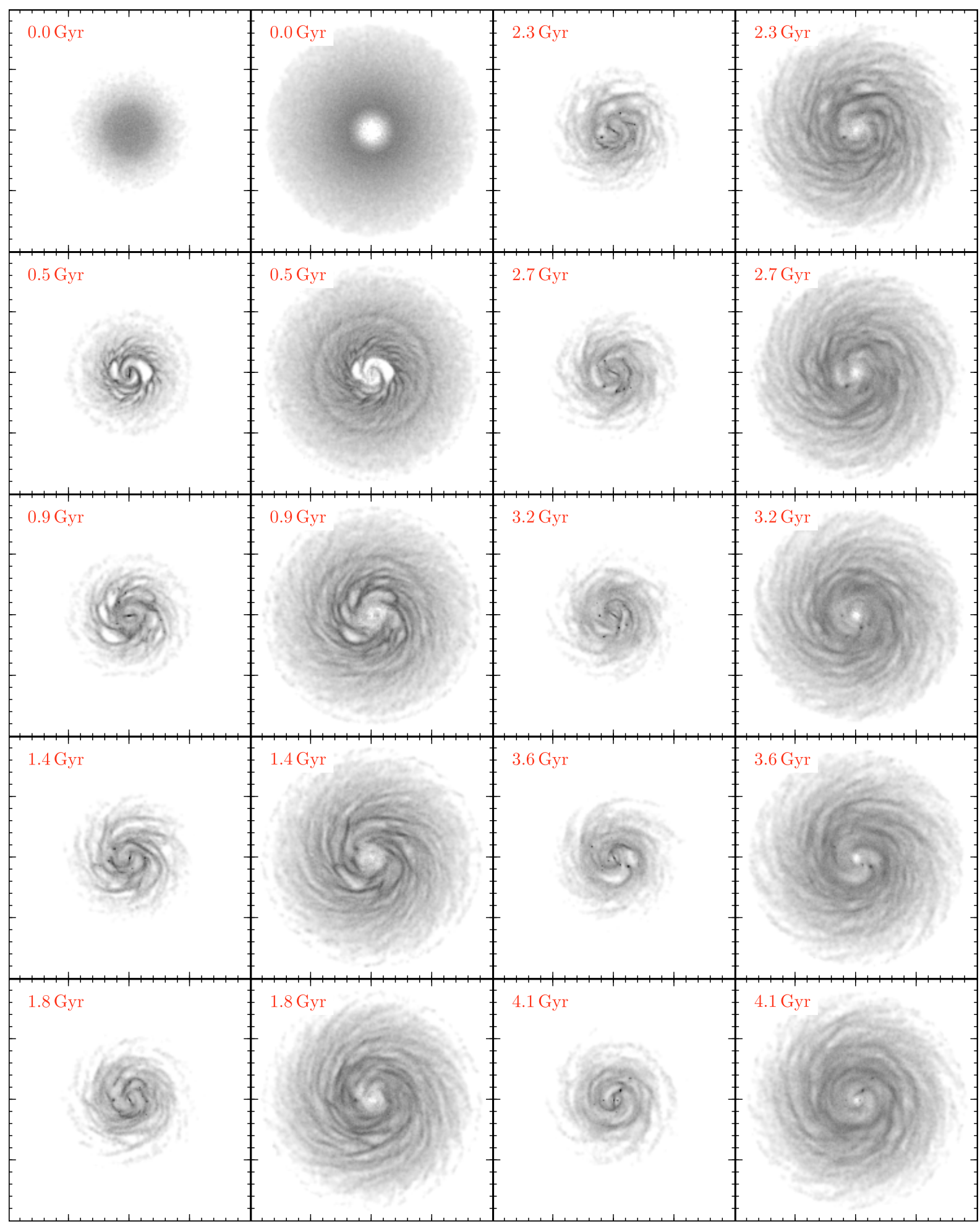

Fig. 15. Evolution of model $N=3$ between 0 and $4.5 \mathrm{Gyr}$. The first and third columns represent the visible gas surface density while the second and fourth represent the dark gas surface density. The box size is $200 \times 200 \mathrm{kpc}$.

simply means that no additional dark matter is expected at the center, even after $4.5 \mathrm{Gyr}$ of evolution.

\subsubsection{Disk stability}

The stability of models $N=3$ and $N=5$ is ensured by the presence of the two partially dynamically decoupled phases. The dark phase has higher velocity dispersions than the visible gas.
The radial velocity dispersions of model $N=3$ are displayed in Fig. 17. Because the dark gas is quasi non-collisional, its radial velocity dispersion is much larger than the one of the visible gas of the reference model (values dropping to $10 \mathrm{~km} \mathrm{~s}^{-1}$ in the outer part.). These higher values ensure the stability of the total baryonic disk. However, our multiphase model fails to reproduce the low velocity dispersions expected in the visible gas. While being clearly decoupled from the dark component, the gas velocity dispersion of model $N=3$ is higher than the one of the reference 


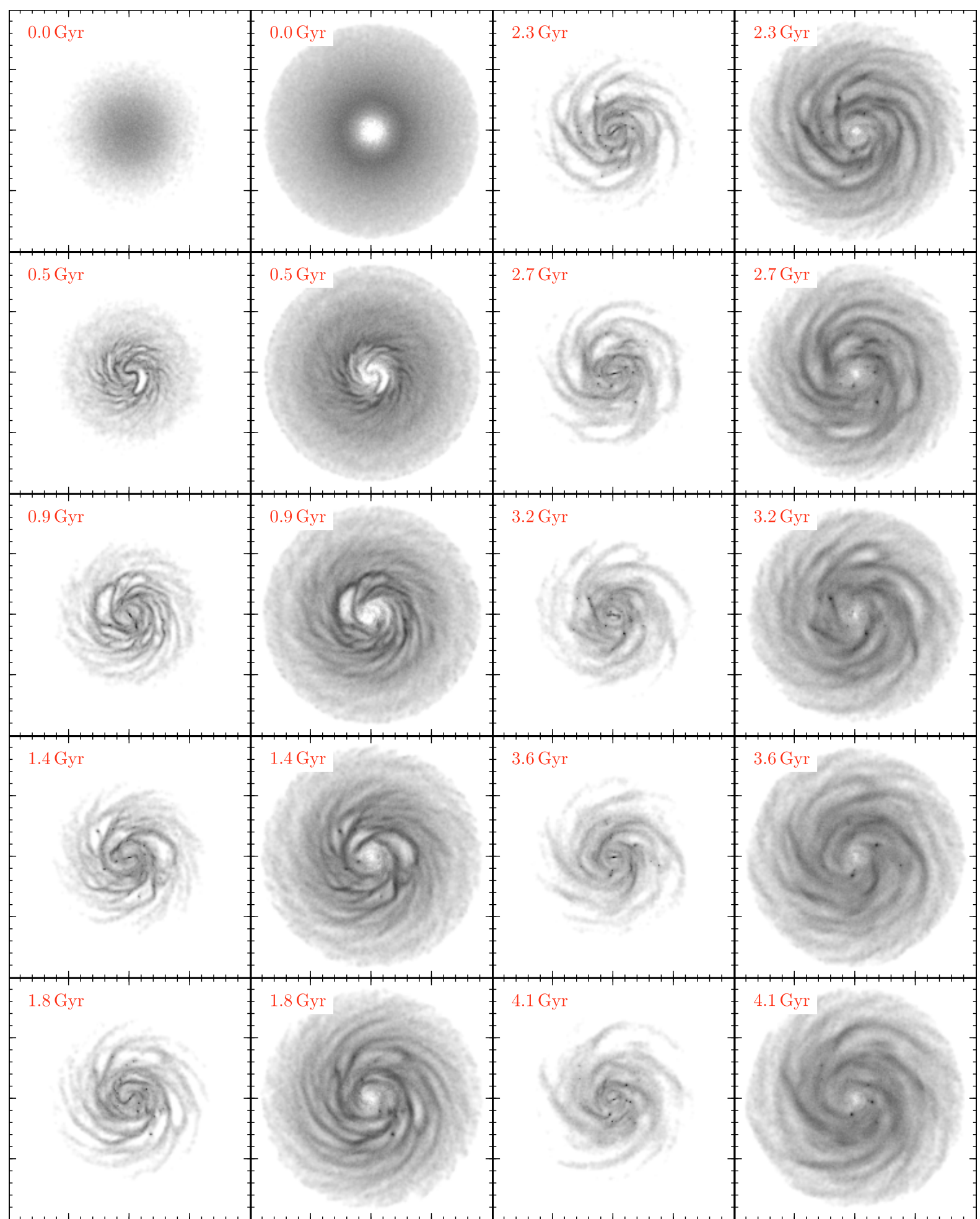

Fig. 16. Evolution of model $N=5$ between 0 and $4.5 \mathrm{Gyr}$. The first and third columns represent the visible gas surface density while the second and fourth represent the dark gas surface density. The box size is $200 \times 200 \mathrm{kpc}$.

model, especially in the outer part. This is the result of the weak coupling due to the cycling between the dark and the visible gas. The model could be improved in the future, by simply increasing the stickiness of particles in the outer part.

As discussed in Appendix B, if one assumes that the dark gas is as dissipative as the visible gas, the resulting velocity dispersions of the total gas is no longer large enough to ensure the disk stability. In that case, the disk breaks and forms small clumps (Fig. B.1).
Since our disk model is made of three dynamically decoupled components (the stellar disk, the visible disk and the dark disk) having distinct velocity dispersions, a precise study of its stability would require to use a multi-component stability criterion. The stability of multi-component galactic disks has been discussed by Jog \& Solomon (1984b,a) and the equivalent of the Savronov-Toomre parameter may be computed (Jog 1996). While this criterion is theoretically valid for an $n$-components system the computational procedure described by Jog (1996) is 


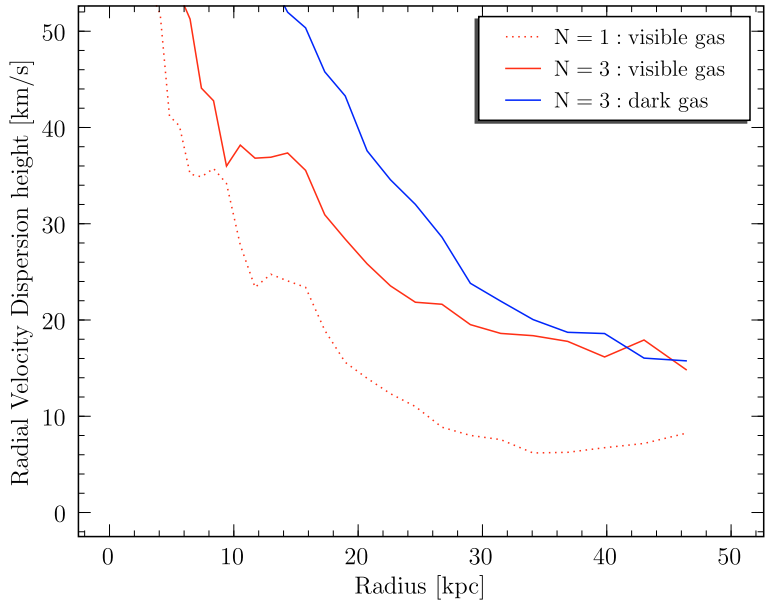

Fig. 17. Radial velocity dispersion at $t=4.5 \mathrm{Gyr}$ of the visible (red) and dark (blue) gas components of model $N=3$. The dotted line corresponds to the radial velocity dispersion of the visible gas of mode $N=1$.

only valid for a two-components system (see also Wang \& Silk 1994; and Elmegreen 1995). We have instead used a SavronovToomre $\left(Q_{1-\mathrm{c}}\right)$ assuming a single component. Formally, this $Q_{1-\mathrm{c}}$ is defined by:

$Q_{1-\mathrm{c}}(R) \simeq \frac{\kappa \sigma_{R, \mathrm{disk}}}{3.36 G \Sigma_{\mathrm{disk}}}$,

where $\Sigma_{\text {disk }}$ and $\sigma_{R \text {,disk }}$ are computed over the visible gas, dark gas and stars particles, while $\kappa$ results from the total potential (including halo dark matter and disk dark baryons). The left panels of Fig. 18 shows the parameter $Q_{1-c}$ for the four models at different times. It is clear that $Q_{1-\mathrm{c}}$ is always larger than 1, explaining the stability observed in Figs. 15 and 16.

\subsection{Spiral structure and swing amplification}

While being larger than 1, the two models with additional baryons are characterized by a nearly constant $Q_{1-\mathrm{c}}$ from 10 to $50 \mathrm{kpc}$, with values between 2 and 3 for model $N=3$ and around 2 for model $N=5$. With these rather low values, the disks are only quasi-stable. Contrasting and open spiral structures are continuously generated up to the end of the dark gas disk at $100 \mathrm{kpc}$. These spirals are thus naturally present in the visible gas up to its end, where its surface density drops, in agreement with most HI observed disks. See for example the impressive case of NGC 6946 (Boomsma et al. 2008).

On the contrary, model $N=1$ has a higher $Q_{1-\mathrm{c}}$, increasing well above 3 for $R>30 \mathrm{kpc}$. Consequently, the outer disk is more stable, preventing the formation of spirals, as observed in Fig. 13. This difference is the key point that allow us to distinguish between models with and without additional baryons.

In order to improve our understanding of the spiral structure, we have computed polar maps $((R-\phi)$-plot $)$ of the visible gas surface density (from $R=0$ to $R=50 \mathrm{kpc}$ ) at different times (Fig. 19). These maps can be, for example, compared to the HI observation in M 83 (Fig. 12 of Crosthwaite et al. 2002). We then performed a Fourier decomposition of those maps, for each radius. The spiral structure can be represented by each dominant azimuthal modes for a given radius, i.e. the mode with the largest amplitude, excluding the $m=0$ mode. Figure 20 displays the amplitude of the dominant azimuthal modes found for each
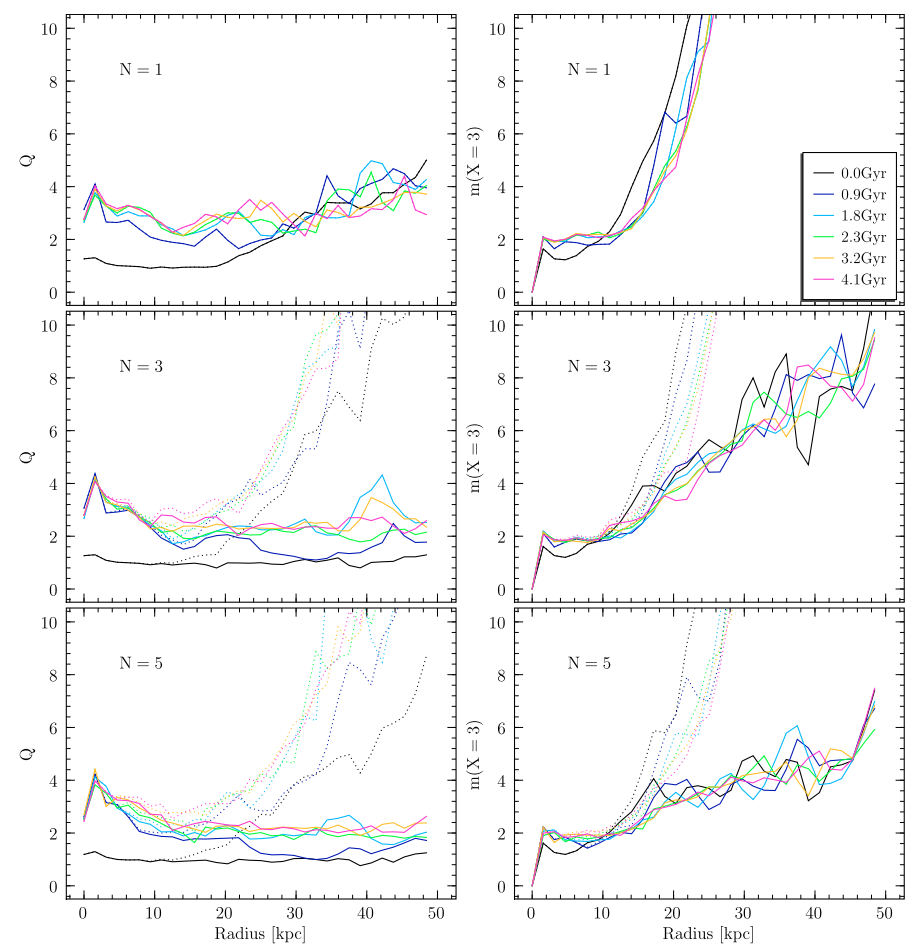

Fig. 18. Stability of models $N=1, N=3$ and $N=5$ at different times $(0,0.9,1.8,2.3,3.2$ and $4.1 \mathrm{Gyr})$. The left column show the Toomre parameter (Eq. (30)). The right column shows the mode corresponding to the swing amplification parameter $X=3$. Modes higher than the latter may be amplified while lower modes are stable. In both columns, the dotted lines correspond to the values computed without taking into account the dark gas. The corresponding color code for all times are given in the upper right panel.

plot of Fig. 19. For a direct comparison between the models, in all plots, the signal amplitude is coded with the same colors. In polar plots, a regular spiral structure of mode $m$ appears as $2 \mathrm{~m}$ inclined parallel lines ${ }^{3}$, where the inclination (that may vary with radius) gives the pitch angle $i$ of the $\operatorname{arm}(i=\pi / 2-\arctan (p)$, where $p$ is the slope of the lines). Such regular features are present for the $N=3$ and $N=5$ models, indicating the presence of rather open spiral arms (large $i$ ) that may be followed up to $50 \mathrm{kpc}$ at nearly all times. The number of arms is not constant and oscillates between 3 and 8 (model $N=3$ ) and 3 and 6 (model $N=5$ ), usually increasing with increasing radius (Bottema 2003). Such large features are not observed for the $N=1$ model. The spiral arms disappear around $R=25 \mathrm{kpc}$.

Spiral arms in a disk may be the result of "swing amplification" (Goldreich \& Lynden-Bell 1965; Julian \& Toomre 1966; Toomre 1981), a mechanism that locally enhances the selfgravity and leads to the amplification of a small perturbation in a differentially rotating structure. The amplification results from the resonance between the epicyclic motions of stars (or gas) and the rate of change of the pitch angle of a density wave, during its conversion from leading to trailing (Binney \& Tremaine 1987). The swing amplification mechanism may be quantified by the parameter $X$, the ratio of the perturbation wavelength and a critical wavelength:

$$
X=\lambda / \lambda_{\mathrm{c}}, \quad \text { where } \quad \lambda_{\mathrm{c}}=\frac{4 \pi^{2} G \Sigma}{\kappa^{2}} .
$$

\footnotetext{
${ }^{3}$ Here, a line is either a maximum or a minimum.
} 


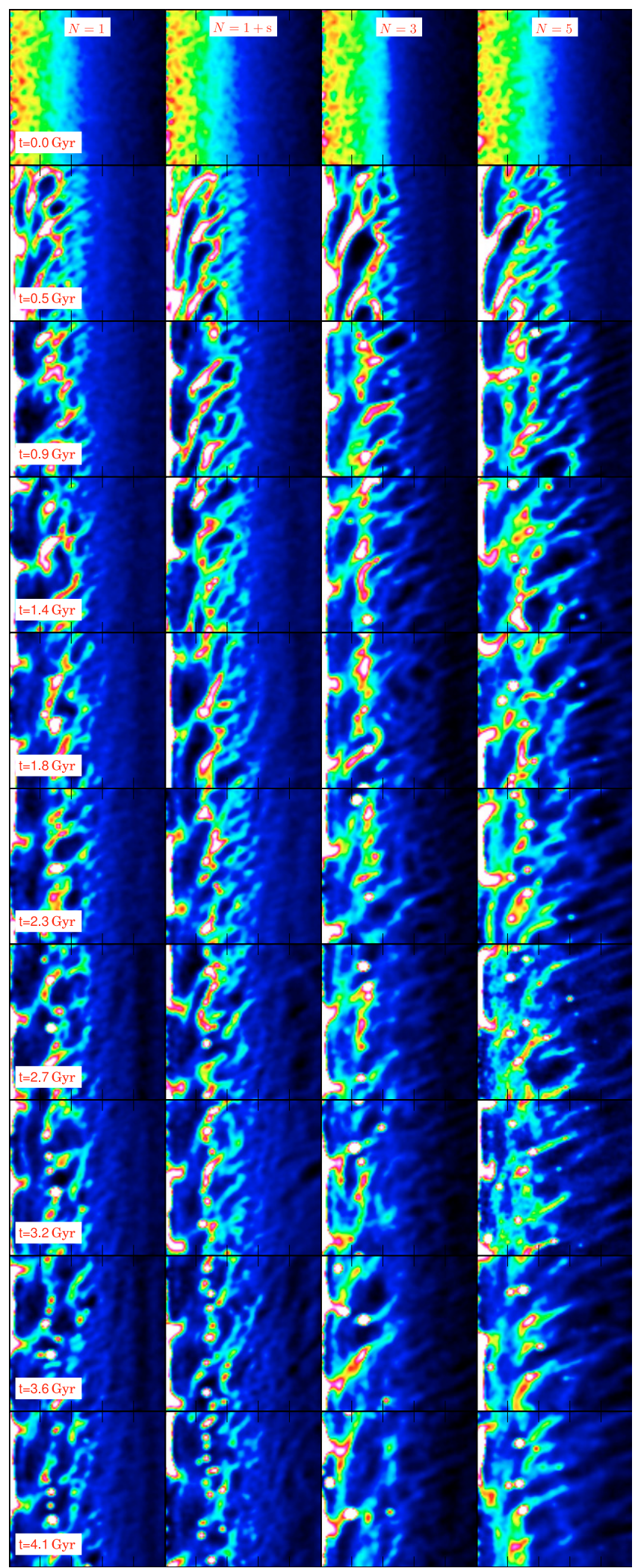

Fig. 19. Evolution of the spiral structure in a $(R-\phi)$-plot of the four models. Each image represents the surface density of the visible gas. The abcissa corresponds to the radius $R$ (from 0 to $50 \mathrm{kpc}$ ) while the ordinate to the azimuthal angle (from 0 to $2 \pi$ ).

Since the wavelength $\lambda$ of a spiral pattern of mode $m$ in a disk is $2 \pi R / m, X$ becomes:

$X(R)=\frac{R \kappa^{2}}{2 \pi G \Sigma m}$
Similarly to the $Q_{1-\mathrm{c}}$ computation, we have computed the swing amplification parameter $X$, taking into account only the surface density of the disk $\left(\Sigma=\Sigma_{\text {disk }}\right)$. As it is too dynamically hot, at first order, the dark matter is not sensitive to the density perturbation and its effect is not included in the computation of $X$. If we assume that the swing amplification is efficient for $X \lesssim 3$ (Toomre 1981; Athanassoula 1984, Fig. 7) ${ }^{4}$, we can reverse Eq. 32 and compute the value of the critical mode $m(X=3)$ :

$m(X=3)=\frac{R \kappa^{2}}{6 \pi G \Sigma_{\mathrm{disk}}}$

The meaning of the critical mode is the following: modes higher than $m(X=3)$ may be amplified by the swing amplification mechanism. On the contrary, the ones above $m(X=3)$ are stable and thus no spiral pattern with $m<m(X=3)$ is observed.

The right panels of Fig. 18 displays the critical modes at different times. For the $N=1$ model, $m(X=3)$ increases very quickly, being above 6 for $R>20 \mathrm{kpc}$. At larger radii, only large $m$ modes, corresponding to noise, may be amplified by the selfgravity of the disk. No spiral pattern is expected, in agreement with Figs. 20 and 13.

When increasing $\Sigma_{\text {disk }}$ by including additional baryons, selfgravity in increased and lower modes may be amplified at larger radius. For example, $m=6$ modes may still be amplified up to $R=35 \mathrm{kpc}$ in model $N=3$ and up to $R=50 \mathrm{kpc}$ in model $N=5$. This explains the patterns present in Fig. 20 and the spiral structure of Figs. 15 and 16.

The dotted lines of Fig. 18 (both in the left and right panels) correspond to the $Q_{1-\mathrm{c}}$ and $m(X=3)$ values as would be deduced by an observer ignoring the presence of the dark gas and taking into account only the visible gas and stars in Eqs. (30) and (33). Clearly, $Q_{1-\mathrm{c}}$ is well above 4 for a radius larger than $20 \mathrm{kpc}$, indicating that the disk should be stable. The $m(X=3)$ parameter implies that modes with low perturbation should be prohibited by the swing amplification theory, in contradiction to the observed spiral structure. This point may explain why spiral structures are present in galaxies where the $Q_{1-c}($ or $X)$ parameter deduced from the visible component is well above 5 (resp. 3 ), as is the case, for example, for the galaxy NGC 2915 (Bureau et al. 1999).

\subsubsection{The effect of the $\Lambda$ CDM satellites}

We can now ask if the perturbations generated by the $\Lambda \mathrm{CDM}$ satellites are able to reproduce the large scale spiral patterns of galaxies, comparable with the ones obtained when including additional baryons.

The effect of the satellites may be seen by comparing the surface density of the visible gas disk of model $N=1$ (Fig. 13) and model $N=1+s$ (Fig. 14). Perturbations in the far outer part of the disk are visible between 2.3 and $4.1 \mathrm{Gyr}$ and appear as asymmetric and winding spiral arms. These perturbations are better traced in Fig. 20, where the same Fourier analysis has been performed for model $N=1+s$. There, the effect of the satellite is already perceptible at $t=1.4 \mathrm{Gyr}$ and $R \sim 30 \mathrm{kpc}$. Between $t=1.4$ and $t=2.3 \mathrm{Gyr}$ an $m=2$ mode develops and disappears afterwards. The large slope of the modes indicates a very small pitch angle. Another $m=2$ mode appears briefly between $t=$ 2.3 and $t=2.7 \mathrm{Gyr}(R>40 \mathrm{kpc})$. In this plot, no clear dominant mode is seen afterwards, between 25 and $50 \mathrm{kpc}$. However, as

\footnotetext{
${ }^{4}$ In fact the exact critical value may vary, depending on the parameter $Q_{1-\mathrm{c}}$ and on the exact velocity curve through the shear parameter $\Gamma=$ $-\frac{r}{\Omega} \frac{\mathrm{d}}{\mathrm{d} r} \Omega$ which in our case is nearly equal to 1 .
} 


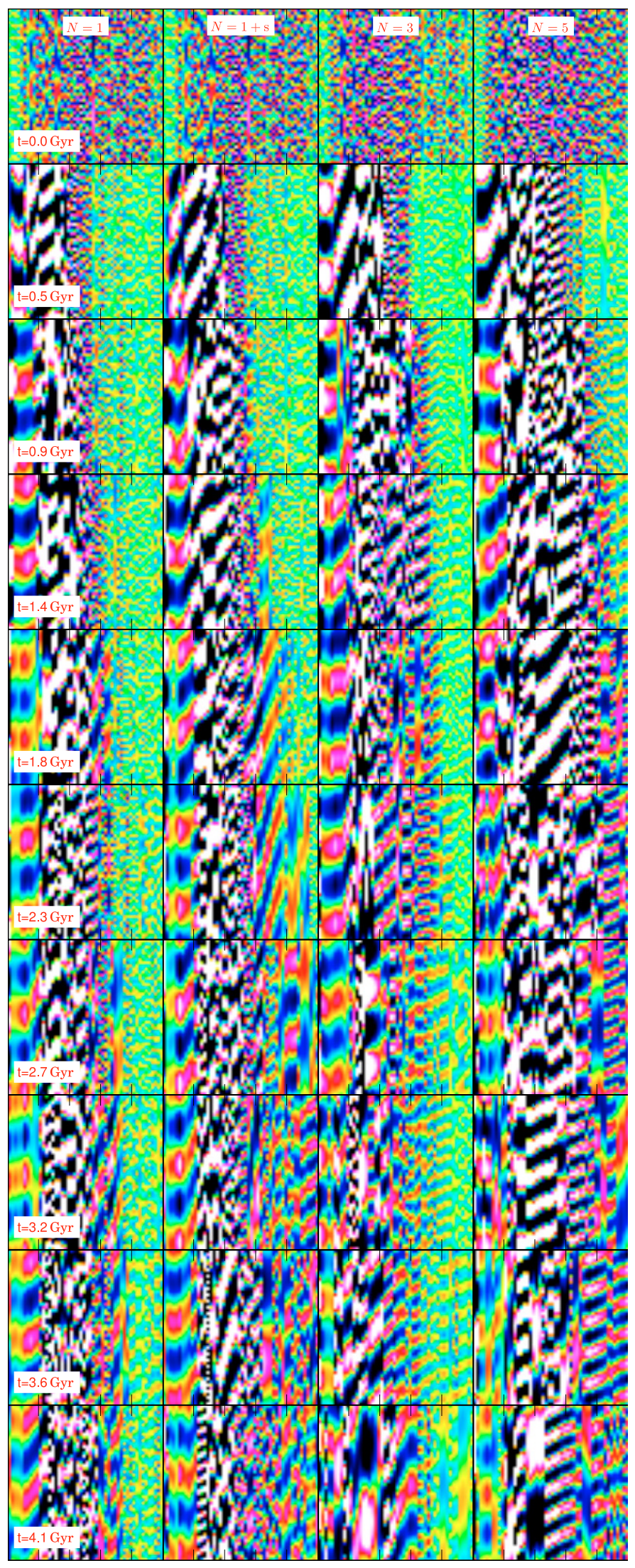

Fig. 20. Evolution of the spiral structure in a $(R-\phi)$-plot of the four models. Each image represent the dominant mode of Fig. 19.

the Fourier decomposition was performed within $R=50 \mathrm{kpc}$, we are missing the spirals observed at larger radii in Fig. 14 at $t=3.6 \mathrm{kpc}$.
The comparison between the spirals resulting from the selfgravity of the disk or the ones formed by satellites perturbations reveals two important differences:

1. As it does not result from a gravity wave, a spiral arm generated by the perturbation due to a satellite is affected by the differential rotation of the disk. Consequently the arm winds quickly (the pitch angle is small) and disappears in a dynamical time (winding problem, see for example Binney \& Tremaine 1987).

2. Spiral patterns generated by the swing amplification are globally continuous along the disk. We have seen for example that the dominant modes slowly decrease outwards. As they result from a local perturbation, asymmetries and strong discontinuities exist in the pattern of spirals induced by satellites. An example is seen in Fig. 20 at $t=2.7 \mathrm{Gyr}$. No dominant mode exists between 25 and $40 \mathrm{kpc}$ while an $m=2$ mode is present at larger radius, disconnected from the rest of the disk.

In conclusion, the $\Lambda \mathrm{CDM}$ satellites are able to perturb a disk and induce spiral arms. However, these arms wind up and disapear quickly. Even if they are periodically induced by satellites, they are unable to reproduce a regular and extended spiral patterns.

According to the theory of spiral structure formation, the fact that $\Lambda \mathrm{CDM}$ induces only localised and decorrelated perturbations is not surprising. Indeed, if satellites act as triggers for disk instabilities, the subsequent amplification of the instabilities leading to the formation of a large scale pattern is determined by the property of the disk itself: its self-gravity (surface density), its velocity curve and its velocity dispersions, the three quantities involved in the $Q_{1-c}$ and $X$ computation. Without the interplay of these essential ingredients, only discontinuous local structures, resulting from the random impact of the satellites on the disk, will be observed.

\section{Discussion and conclusion}

Using a new self-consistent $N$-body multiphase model, we have shown that the hypothesis where galaxies are assumed to have an additional dark baryonic component is in agreement with spiral galaxy properties. The key element of the model is to assume that the ISM is composed out of two partially dynamically decoupled phases, the observed dissipative gas phase and a dark, very cold, clumpy and weakly collisional phase. Motivations for invoking a similar component made of cold invisible gas, like the disk-halo conspiracy, the HI-dark matter proportionality, extreme scattering and microlensing events, the hydrostatic equilibrium of the Galaxy or the survival of small molecular structures without shielding, have been given many times in the literature (e.g., Pfenniger \& Combes 1994; Henriksen \& Widrow 1995; Gerhard \& Silk 1996; Walker \& Wardle 1998; Kalberla \& Kerp 1998; Kerins et al. 2002; Heithausen 2002, 2004).

An original scheme, designed to overcome numerical limitations, has been proposed to compute the cycling between these two phases when subject to heating and cooling. From this scheme, we have shown that realistic self-consistent $N$-body models of spiral galaxies containing an additional baryonic dark matter content may be constructed. These models share similar observational properties with classical CDM disks, like the rotation curve and visible gas surface density and in that sense, are observationally similar.

The main result of this work is that, despite having more mass in the disk, these systems are globally stable, the stability 
being ensured by the larger velocity dispersion of the dark gas that dominates the gravity in the outer part of the disk. In addition, the enhanced self-gravity of these disks, due to the presence of the dark clumpy gas, makes them more prone to form spirals extending up to $100 \mathrm{kpc}$ in the dark gas. The spiral structure is revealed by the $\mathrm{HI}$ that acts as a tracer of the dark gas, up to the radius where its surface density becomes so small that the hydrogen is fully ionized and hardly observable. This gives a natural solution of the numerous observations of $\mathrm{HI}$ unstable disks that are difficult to explain by the self-gravity of the HI disk alone (see for example Bureau et al. 1999; Masset \& Bureau 2003).

Depending on the theory of spiral structure (the local stability of differentially rotating disks and the swing amplification mechanism) our results depend on the three quantities involved in the Toomre parameters: the disk self-gravity by its surface density, its rotation curve and its radial velocity dispersion. Consequently, these results will not be affected by a different parametrisation of the stellar disk or the dark halo, as long as a similar rotation curve is reproduced.

We have seen that in our models the velocity dispersion of the collisionless or weakly collisional components increases with time, similarly to what stars are known to do in the Milky Way in the so-called Wielen's diffusion. This is fully expected from our general understanding of the evolution of selfgravitating disks of collisionless particles, where the effects of spiral arms and especially a bar are sufficient to cause such a radial heating. A more detailed discussion and references are given in Sellwood \& Binney (2002).

While the cold gas phase is probably weakly collisional, we have treated it as strictly collisionless. How will the spiral morphology then be modified by introducing a weak dissipation in this component? In Appendix B we present the case where the dark component is as dissipative as the observed gas. In this extreme case, the disk is strongly unstable and fragments as a consequence of the Jeans instability. At some point, decreasing the dissipation of the dark gas strengthens the global stability, while reinforcing the spiral arm contrast. The dynamical coupling of the two phases through the cycling is as important as the dissipation. A very short transition timescale $\tau$ leads to a strong coupling. Additional simulations show that in that case, the velocity dispersions of the two components are nearly similar, the one of the observed gas being higher. Consequently, the spiral structures are less contrasting. On the contrary, if the two phases are decoupled, the observed gas velocity dispersion is smaller, and the arm contrast higher. In both cases however, the large scale spiral pattern remains similar.

Additional simulations have investigated the effect of $\Lambda C D M$ substructures on the outer HI disk. Satellites only generate local, winding and short-lived arms. Because the HI disk is not sufficiently self-gravitating, its response is too small to amplify the perturbations. In that sense, we confirm the results of Dubinski et al. (2008). The tidal effects of the satellites are generally small and so are not responsible for large scale spiral patterns.

If models of galactic disks with additional dark baryons are in agreement with observations, we must ask whether they are in agreement with the $\Lambda \mathrm{CDM}$ scenario. From the cosmic baryon budget (Fukugita \& Peebles 2004), we know that nearly $60 \%$ of the baryons predicted from primordial nucleosynthesis are not observed. Multiplying the galactic baryon content (about $8 \%$ ) by a factor of 2, as it is assumed in our most massive $N=5$ model, will still be in agreement with the baryon budget. From a galactic point of view, multiplying the baryon content by a factor 2 is well inside the observed uncertainties. For a circular velocity curve of about $250 \mathrm{~km} \mathrm{~s}^{-1}$ typical of our models, the baryon content varies by more than a factor of 5 (see Fig. 1 of Mayer \& Moore 2004). Assuming a corresponding virial mass of $10^{12} M_{\odot}$ the cosmic baryon fraction may vary around 10 and $50 \%$. With a baryonic fraction of $29 \%$, our $N=5$ model corresponds to a large but realistic value.

Numerical simulations of the formation of large scale structures predict that the "missing baryons" reside in a warm-hot gas phase in the over-dense cosmic filaments (Cen \& Ostriker 1999, 2006). However, there are now also signs of accretion of cold gas during the build up of galactic disks (Kereš et al. 2005). As discussed in Sect. 2, a low density resolution leads to an underestimate of the gas cooling, missing over-dense regions present in an inhomogeneous ISM, where the cooling time of the gas is very short. Since the assumed amount of dark baryons is not in contradiction with the cosmic baryon budget, we need to wait for future works to see if the correct treatment of inhomogeneous ISM, including shocks, is able to cool $10 \%$ of the "missing baryons" during the hierarchical structure formation, making the present model consistent with the $\Lambda \mathrm{CDM}$ scenario.

In a companion paper, we have shown that our model also explains the puzzling presence of dark matter in the collisional debris from galaxies (Bournaud et al. 2007).

The remaining questions are obviously why the cold dark phase is invisible and what the physics ruling it might consist of. Since star formation is also a poorly understood process, but we know that stars do form from the coldest observable molecular gas, the question of why stars are not supposed to form in the coldest form of our invoked dark gas remains open. Suggestions of why star formation may not always start in cold gas have been given in Pfenniger \& Combes (1994). Essentially, if molecular gas fragmentation goes down to sub-stellar mass clumps staying cold, these clumps are unable to free nuclear energy and become stars. Since they are close to the $3 \mathrm{~K}$ background, they almost do not radiate. Because of their negative specific capacity, such self-gravitating clumps do not increase their temperature when subject to external heating, but stay cold while evaporating, a sometimes overlooked feature of self-gravity. Many earlier works have discussed these issues, for example how cold molecular hydrogem may clump in dense structures of solar system size but stay undetected (Combes \& Pfenniger 1997; Pfenniger \& Combes 1994; Pfenniger 2004). However, much more work remains to understand the precise physics of very cold gas $(T<10 \mathrm{~K})$ in low excitation regions, like the outer galactic disks, but also in planetary nebulae and star forming regions where observed dense cold gas clumps self-shield from outer radiation and reach sub- $10 \mathrm{~K}$ temperatures. It is generally known that $\mathrm{H}_{2}$ condenses in solid form even at interstellar pressures at temperatures close to the $3 \mathrm{~K}$ cosmic background. A phase transition means that a richer physics must be expected. But since $\mathrm{H}_{2}$ is usually mixed with about $10 \%$ of helium in mass, it was for many years not clear how to describe the equation of state of this mixture in such conditions. Recently, Safa \& Pfenniger (2008) succeeded in describing this mixture for astrophysically interesting conditions with chemo-physical methods, reproducing its main characteristics like the critical point and the condensation curve, as well as predicting the conditions of He$\mathrm{H}_{2}$ separation. Work is in progress to apply these results to the cold interstellar gas.

Acknowledgements. It is a pleasure to thank Chandra Jog for discussions on the stability of multi-components systems and Jerry Sellwood for motivating us to perform Fourier analysis. The simulations have been run on the Horizon mini grid and the Horizon meso-machine in Paris, as well as on the Regor cluster at the Geneva Observatory. Data reduction and galaxy maps have been performed 
using the parallelized Python pNbody package (see http://obswww.unige . $\mathrm{ch} / \sim \mathrm{revaz} / \mathrm{pNbody} /$ ). This work was supported by the Swiss National Science Foundation and by the French Centre National Pour la Recherche Scientifique (CNRS).

\section{Appendix A: The Miyamoto-Nagai potential: another parametrization}

The Miyamoto-Nagai potential (Miyamoto \& Nagai 1975) in cylindrical coordinates is usually written as:

$\Phi^{\mathrm{MN}}(R, z)=-\frac{G M}{\sqrt{R^{2}+\left(a+\sqrt{z^{2}+b^{2}}\right)^{2}}}$,

where $M$ is the total mass and $a$ and $b$ are two parameters. It is useful to parametrize the Miyamoto-Nagai potential, introducing the horizontal scale length $h_{R}$ and the vertical scale length $h_{z}$ defined by:

$h_{R}=a+b$ and $h_{z}=b$.

The potential then becomes:

$\Phi_{\left(M, h_{z}, h_{R}\right)}^{\mathrm{MN}}(R, z)=-\frac{G M}{\sqrt{R^{2}+\left(h_{R}-h_{z}+\sqrt{z^{2}+h_{z}^{2}}\right)^{2}}}$.

Using this parametrization, the circular velocity curve is written as:

$v_{\mathrm{c}}^{2}=R \frac{\partial}{\partial R} \Phi^{\mathrm{MN}}(R, 0)=\frac{G M R^{2}}{\left[R^{2}+h_{R}^{2}\right]^{3 / 2}}$,

which is independent of the vertical scale length $h_{z}$. This means that any Miyamoto-Nagai model with mass $M$ and horizontal scale length $h_{R}$ will fit the same rotation curve. One may also recognize in Eq. (A.4) the familiar velocity curve of the Plummer model, the latter being a subclass of the Miyamoto-Nagai model, with $a=0$ and $b=h_{R}$.

A corollary of Eq. (A.4) is that the rotation curve of any Miyamoto-Nagai model can be reproduced by a linear combination of two or more Miyamoto-Nagai models, having different scale heights and appropriate masses. For example, the potential $\Phi_{M}^{\prime}(R, z)$ defined by

$\Phi_{M}^{\prime}(R, z)=f \Phi_{\left(M, h_{z}^{\prime}, h_{R}\right)}^{\mathrm{MN}}(R, z)+(1-f) \Phi_{\left(M, h_{z}, h_{R}\right)}^{\mathrm{MN}}(R, z)$,

where $f \in[0,1]$ and $h_{z}^{\prime} \neq h_{z}$, will have the same velocity curve as $\Phi_{\left(M, h_{z}, h_{R}\right)}^{\mathrm{MN}}(R, z)$.

\section{Appendix B: Evolution of model $\mathbf{N}=\mathbf{3}^{\prime}$}

In this appendix we present the evolution of model $N=3^{\prime}$, where all the gas (visible and dark) is assumed to be dissipative, computed with the sticky particle scheme (see Sect. 2.3). The sticky parameters used are similar to the ones used for the visible gas in model $N=3$ and $N=5$. The evolution shows that at the beginning $(t=0-t=0.8 \mathrm{Gyr})$ the strongest dissipation reinforces the spiral constant in the central part of the galaxy. But after $1 \mathrm{Gyr}$ the inner disk, which is dynamically very cold, becomes strongly unstable. The spirals break into several dense clumps and the disk does not look like a real galaxy. This effect is even stronger for an $N=5$ model, which is not displayed here. This test shows that the dark matter in the disk cannot be as dissipative as the visible disk. Otherwise, the disk is unstable and fragments in less than a dynamical time.

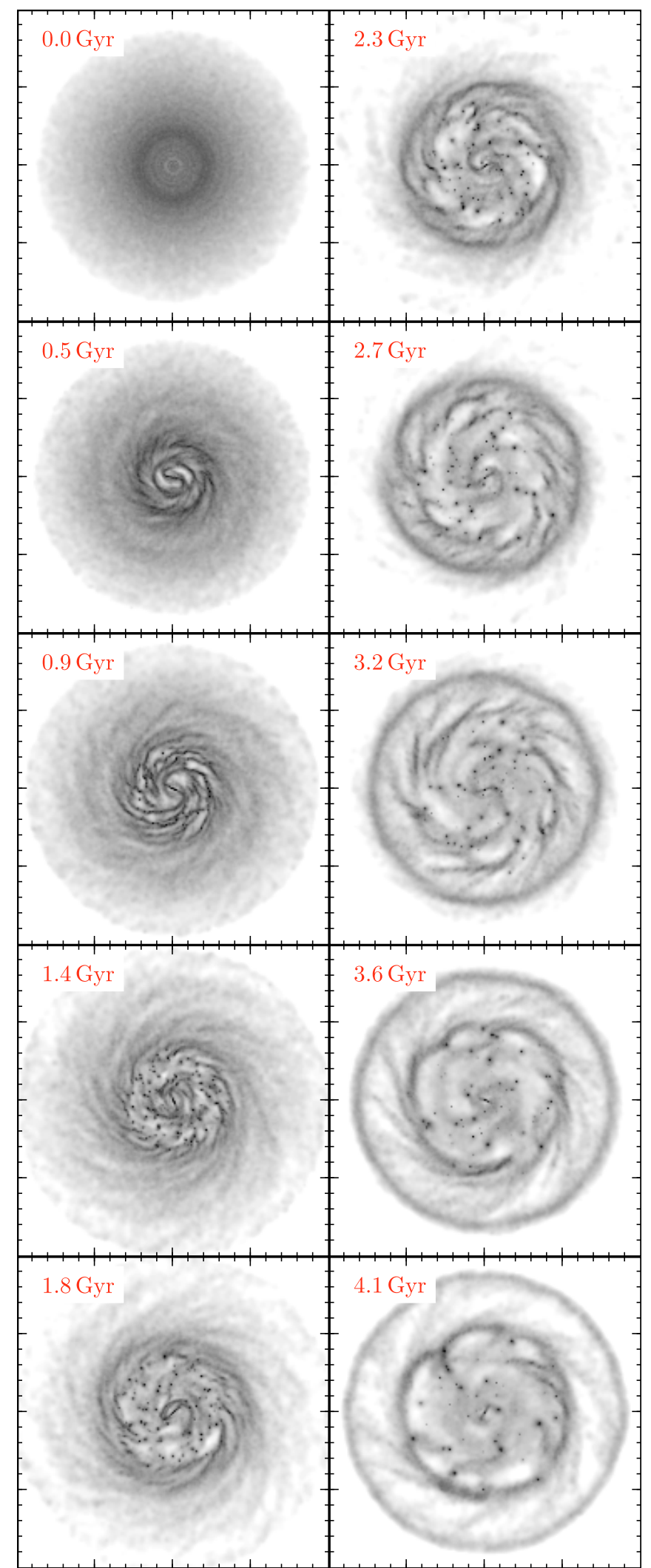

Fig. B.1. Evolution of model $N=3^{\prime}$ where the all the gas (visible and dark) is assumed to be dissipative. Each image represents the surface density of the visible gas. The box size is $200 \times 200 \mathrm{kpc}$.

\section{References}

Appleton, P. N., Xu, K. C., Reach, W., et al. 2006, ApJ, 639, L51 Athanassoula, E. 1984, Phys. Rep., 114, 321 
Begum, A., Chengalur, J. N., Karachentsev, I. D., \& Sharina, M. E. 2008, MNRAS, 386, 138

Binney, J., \& Tremaine, S. 1987, Galactic dynamics (Princeton, NJ: Princeton University Press)

Bissantz, N., \& Gerhard, O. 2002, MNRAS, 330, 591

Blais-Ouellette, S., Amram, P., \& Carignan, C. 2001, AJ, 121, 1952

Boomsma, R., Oosterloo, T. A., Fraternali, F., van der Hulst, J. M., \& Sancisi, R. 2008, ArXiv e-prints, 807

Bosma, A. 1978, Ph.D. Thesis, Groningen Univ.

Bosma, A. 1981, AJ, 86, 1825

Bottema, R. 2003, MNRAS, 344, 358

Boulanger, F., Maillard, J. P., Appleton, P., et al. 2008, Experimental Astronomy, 20

Bournaud, F., \& Combes, F. 2002, A\&A, 392, 83

Bournaud, F., Duc, P.-A., Brinks, E., et al. 2007, Science, 316, 1166

Brahic, A. 1977, A\&A, 54, 895

Broeils, A. H. 1992, Ph.D. Thesis, Univ. Groningen

Bureau, M., Freeman, K. C., Pfitzner, D. W., \& Meurer, G. R. 1999, AJ, 118, 2158

Carignan, C., Charbonneau, P., Boulanger, F., \& Viallefond, F. 1990, A\&A, 234, 43

Cen, R., \& Ostriker, J. P. 1999, ApJ, 514, 1

Cen, R., \& Ostriker, J. P. 2006, ApJ, 650, 560

Combes, F., \& Gerin, M. 1985, A\&A, 150, 327

Combes, F., \& Pfenniger, D. 1997, A\&A, 327, 453

Crosthwaite, L. P., Turner, J. L., Buchholz, L., Ho, P. T. P., \& Martin, R. N. 2002, AJ, 123, 1892

Cuillandre, J.-C., Lequeux, J., Allen, R. J., Mellier, Y., \& Bertin, E. 2001, ApJ, 554,190

de Blok, W. J. G. 2005, ApJ, 634, 227

de Blok, W. J. G., \& Bosma, A. 2002, A\&A, 385, 816

de Blok, W. J. G., \& Walter, F. 2003, MNRAS, 341, L39

de Blok, W. J. G., Walter, F., Brinks, E., et al. 2008, AJ, 136, 2648

Draine, B. T. 1978, ApJS, 36, 595

Draine, B. T. 1998, ApJ, 509, L41

Dubinski, J., Gauthier, J.-R., Widrow, L., \& Nickerson, S. 2008, in ASP Conf. Ser. 396, ed. J. G. Funes \& E. M. Corsini, 321

Egami, E., Rieke, G. H., Fadda, D., \& Hines, D. C. 2006, ApJ, 652, L21

Elmegreen, B. G. 1995, MNRAS, 275, 944

Elmegreen, B. G. 1997, in New extragalactic perspectives in the new South Africa (Kluwer, 1996), 117, 467

Ferguson, A. M. N., Wyse, R. F. G., Gallagher, J. S., \& Hunter, D. A. 1998, ApJ, 506, L19

Font, A. S., Navarro, J. F., Stadel, J., \& Quinn, T. 2001, ApJ, 563, L1

Fukugita, M., \& Peebles, P. J. E. 2004, ApJ, 616, 643

Fux, R. 2005, A\&A, 430, 853

Gao, L., White, S. D. M., Jenkins, A., Stoehr, F., \& Springel, V. 2004, MNRAS, 355,819

Gauthier, J.-R., Dubinski, J., \& Widrow, L. M. 2006, ApJ, 653, 1180

Gentile, G., Salucci, P., Klein, U., Vergani, D., \& Kalberla, P. 2004, MNRAS, 351,903

Gentile, G., Burkert, A., Salucci, P., Klein, U., \& Walter, F. 2005, ApJ, 634, L145

Gerhard, O. 2006, in EAS Publications Series, ed. G. A. Mamon, F. Combes, C. Deffayet, \& B. Fort, EAS Publ. Ser., 20, 89

Gerhard, O., \& Silk, J. 1996, ApJ, 472, 34

Goldreich, P., \& Lynden-Bell, D. 1965, MNRAS, 130, 125

Goldsmith, D. W., Habing, H. J., \& Field, G. B. 1969, ApJ, 158, 173

Grenier, I. A., Casandjian, J.-M., \& Terrier, R. 2005, Science, 307, 1292

Habing, H. J. 1968, Bull. Astron. Inst. Netherlands, 19, 421

Harfst, S., Theis, C., \& Hensler, G. 2006, A\&A, 449, 509

Heithausen, A. 2002, A\&A, 393, L41

Heithausen, A. 2004, ApJ, 606, L13

Henriksen, R. N., \& Widrow, L. M. 1995, ApJ, 441, 70

Hernquist, L. 1993, ApJS, 86, 389

Hoekstra, H., van Albada, T. S., \& Sancisi, R. 2001, MNRAS, 323, 453
Jiang, I.-G., \& Binney, J. 1999, MNRAS, 303, L7

Jog, C. J. 1996, MNRAS, 278, 209

Jog, C. J., \& Solomon, P. M. 1984a, ApJ, 276, 127

Jog, C. J., \& Solomon, P. M. 1984b, ApJ, 276, 114

Johnston, K. V., Law, D. R., \& Majewski, S. R. 2005, ApJ, 619, 800

Julian, W. H., \& Toomre, A. 1966, ApJ, 146, 810

Kalberla, P. M. W. 2003, ApJ, 588, 805

Kalberla, P. M. W. 2004, Ap\&SS, 289, 239

Kalberla, P. M. W., \& Kerp, J. 1998, A\&A, 339, 745

Kalberla, P. M. W., Dedes, L., Kerp, J., \& Haud, U. 2007, A\&A, 469, 511

Katz, N., Weinberg, D. H., \& Hernquist, L. 1996, ApJS, 105, 19

Kaufmann, T., Mayer, L., Wadsley, J., Stadel, J., \& Moore, B. 2007, MNRAS, 375,53

Kereš, D., Katz, N., Weinberg, D. H., \& Davé, R. 2005, MNRAS, 363, 2

Kerins, E., Binney, J., \& Silk, J. 2002, MNRAS, 332, L29

Klypin, A., Kravtsov, A. V., Valenzuela, O., \& Prada, F. 1999, ApJ, 522, 82

Lelièvre, M., \& Roy, J.-R. 2000, AJ, 120, 1306

Maio, U., Dolag, K., Ciardi, B., \& Tornatore, L. 2007, MNRAS, 379, 963

Masset, F. S., \& Bureau, M. 2003, ApJ, 586, 152

Mayer, L., \& Moore, B. 2004, MNRAS, 354, 477

Mayer, L., Governato, F., \& Kaufmann, T. 2008, Adv. Sci. Lett., 1, 7

Merlin, E., \& Chiosi, C. 2007, A\&A, 473, 733

Miyamoto, M., \& Nagai, R. 1975, PASJ, 27, 533

Moore, B., Ghigna, S., Governato, F., et al. 1999, ApJ, 524, L19

Navarro, J. F., \& Benz, W. 1991, ApJ, 380, 320

Navarro, J. F., \& Steinmetz, M. 1997, ApJ, 478, 13

Ogle, P., Antonucci, R., Appleton, P. N., \& Whysong, D. 2007, ApJ, 668, 699

Papadopoulos, P. P., Thi, W.-F., \& Viti, S. 2002, ApJ, 579, 270

Pfenniger, D. 2004, in Baryons in Dark Matter Halos, ed. R. Dettmar, U. Klein, \& P. Salucci

Pfenniger, D., \& Combes, F. 1994, A\&A, 285, 94

Pfenniger, D., \& Revaz, Y. 2005, A\&A, 431, 511

Pfenniger, D., Combes, F., \& Martinet, L. 1994, A\&A, 285, 79

Reach, W. T., Franz, B. A., Weiland, J. L., et al. 1995, Nature, 374, 521

Reshetnikov, V., \& Combes, F. 1999, A\&AS, 138, 101

Revaz, Y., \& Pfenniger, D. 2004, A\&A, 425, 67

Revaz, Y., \& Pfenniger, D. 2007, a New Scenario for the Origin of Galacticwarps, Island Universes - Structure and Evolution of Disk Galaxies, 149

Safa, Y., \& Pfenniger, D. 2008, Eur. Phys. J. B, 66, 337

Sánchez-Saavedra, M. L., Battaner, E., Guijarro, A., López-Corredoira, M., \& Castro-Rodríguez, N. 2003, A\&A, 399, 457

Schwarz, M. P. 1981, ApJ, 247, 77

Seiden, P. E., Schulman, L. S., \& Elmegreen, B. G. 1984, ApJ, 282, 95

Sellwood, J. A., \& Binney, J. J. 2002, MNRAS, 336, 785

Semelin, B., \& Combes, F. 2002, A\&A, 388, 826

Shen, J., \& Sellwood, J. A. 2006, MNRAS, 370, 2

Smith, D. A., Allen, R. J., Bohlin, R. C., Nicholson, N., \& Stecher, T. P. 2000, ApJ, 538, 608

Spano, M., Marcelin, M., Amram, P., et al. 2008, MNRAS, 383, 297

Spekkens, K., Giovanelli, R., \& Haynes, M. P. 2005, AJ, 129, 2119

Springel, V. 2005, MNRAS, 364, 1105

Springel, V., Frenk, C. S., \& White, S. D. M. 2006, Nature, 440, 1137

Strigari, L. E., Bullock, J. S., Kaplinghat, M., et al. 2007, ApJ, 669, 676

Swaters, R. A., Madore, B. F., van den Bosch, F. C., \& Balcells, M. 2003, ApJ, 583,732

Thilker, D. A., Bianchi, L., Boissier, S., et al. 2005, ApJ, 619, L79

Toomre, A. 1981, in Structure and Evolution of Normal Galaxies, ed. S. M. Fall, \& D. Lynden-Bell, 111

van den Bosch, F. C. 2001, MNRAS, 327, 1334

Walker, M., \& Wardle, M. 1998, ApJ, 498, L125

Wang, B., \& Silk, J. 1994, ApJ, 427, 759

Watson, W. D. 1972, ApJ, 176, 103

Weinberg, M. D., \& Blitz, L. 2006, ApJ, 641, L33 